\title{
Alternating Direction Method of Multipliers \\ for Truss Topology Optimization with Limited Number of Nodes: A Cardinality-Constrained Second-Order Cone Programming Approach
}

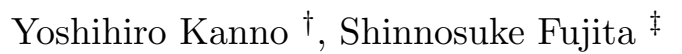

\begin{abstract}
This paper addresses the compliance minimization of a truss, where the number of available nodes is limited. It is shown that this optimization problem can be recast as a second-order cone programming with a cardinality constraint. We propose a simple heuristic based on the alternative direction method of multipliers. The efficiency of the proposed method is compared with a global optimization approach based on mixed-integer second-order cone programming. Numerical experiments demonstrate that the proposed method often finds a solution having a good objective value with small computational cost.
\end{abstract}

\section{Keywords}

Topology optimization; truss optimization; manufacturability; alternating direction method of multipliers; cardinality-constrained second-order cone programming; mixed-integer second-order cone programming.

\section{Introduction}

It is common to use the ground structure method [8, 31, 54] for truss topology optimization, where the cross-sectional areas of the truss members are treated as design variables to be optimized. Particularly, the compliance minimization with the continuous design variables is convex [8, 44], and can be solved efficiently. An optimal solution of this problem often consists of too many members (including ones that are too thin) connected by many nodes 1 and, hence, is regarded as too complex a design from the viewpoint of practical manufacturability. Also, the fabrication cost of a truss usually increases as the number of nodes increases. To obtain a practically acceptable truss design, Asadpoure et al. [5] proposed to minimize the weighted sum of the structural weight and the fabrication cost related to the number of members. In this method, the number of members is approximated by using a regularized Heaviside step function. Torii et al. [55] used the same approach to take into account the number of nodes. In this paper, we consider the compliance minimization of a truss subjected to the explicit upper bound constraint on the number of nodes.

The number of nodes in structural optimization has also been discussed in the layout optimization of trusses. In the classical layout optimization, we minimize the total weight of the members when the allowable stress is specified. When the potential locations of nodes of a truss are not limited, the optimal solution becomes a so-called truss-like continuum with infinitely many nodes [25, 41]. Prager [48, 49] showed that, by adding the weight of the nodes to the objective function,

\footnotetext{
${ }^{\dagger}$ Corresponding author. Mathematics and Informatics Center, The University of Tokyo, Hongo 7-3-1, Tokyo 1138656, Japan. E-mail: kanno@mi.u-tokyo.ac.jp.

${ }^{\ddagger}$ Laboratory for Future Interdisciplinary Research of Science and Technology, Institute of Innovative Research, Tokyo Institute of Technology, Nagatsuta 4259, Yokohama 226-8503, Japan. E-mail: fujita.s.ag@m.titech.ac.jp.

${ }^{1}$ See, for example, Figure 6 in Section 6
} 
we can obtain an optimal solution with a finite number of nodes. To avoid complex truss design, Parkes [46] proposed to introduce modification of member lengths such that, at each node, a constant is added to the length of each member connected to the node. As a post-processing step for this method, He and Gilbert [23] proposed to make use of the geometry optimization. Similarly, Mazurek et al. 39] defined a so-called performance index, by using the member lengths and the axial forces, to assess the cost of a structure; see also [38. The number of nodes in a truss is not specified explicitly in the methods in the literature [5, 23, 38, 39, 46, 55] cited above.

In this paper, based on the ground structure method we deal with the compliance minimization problem of a truss subjected to the upper bound constraint on the number of nodes (i.e., the cardinality constraint on the set of nodes). This design optimization problem essentially consists of two decisions: We first select a set of nodes, satisfying the cardinality constraint, among the candidate nodes in a ground structure, and next find the optimal cross-sectional areas of the members connected to the selected nodes. The first decision gives combinatorial attribute to the design optimization problem. In this paper, we show that this optimization problem can be recast as mixed-integer second-order cone programming (MISOCP); see Section 3.2. Since an SOCP problem can be solved efficiently with a primal-dual interior-point method [3, 7], we can compute a global optimal solution of an MISOCP problem with, e.g., a branch-and-bound method. Several software packages are available for this purpose [2, 22]. However, due to its large computational cost, the MISOCP approach can be applied only to small- to medium-size truss optimization problems. The reader may refer to [9, 42] for applications of MISOCP to variable selection in statistics, and [27 29, 32] for applications in structural optimization.

The number of nonzero components of a real vector is called the $\ell_{0}$-norm of the vector 2 An upper bound constraint on the $\ell_{0}$-norm of a vector, i.e., the $\ell_{0}$-norm constraint, is also called the cardinality constraint (i.e., the upper bound constraint on the cardinality of the support of the vector). The cardinality constraint, as well as the $\ell_{0}$-norm minimization, frequently appears in diverse fields including variable selection in statistics, image processing, compressed sensing, and portfolio selection [9, 11-14, 17, 19, 33, 43, 57. An application of the $\ell_{0}$-minimization to structural design generating link mechanisms can be found in [45]. In this paper, we show that the truss topology optimization with the limited number of nodes can be formulated as cardinality-constrained SOCP; see Section 3.1 .

The alternating direction method of multipliers (ADMM) is an algorithm for convex optimization [10]. For various nonconvex optimization problems, it is known that ADMM can often serve as a simple but powerful heuristic [15, 16, 18, 26, 36, 53]. This motivates us to develop a simple heuristic based on ADMM, to find approximate solutions to the truss topology optimization with limited number of nodes. The proposed method might be expected to find a local optimal solution having the reasonable objective value with small computational cost. In control theory, ADMM has been used for various sparsity-promoting optimal control method, including design of sparse feedback gains [34, sparse output feedback [4, and a sparse gain matrix for the extended Kalman filter [37].

The paper is organized as follows: Section 2 provides an overview of necessary backgrounds of ADMM. Section 3 formulates the truss topology optimization problem with the limited number of

\footnotetext{
${ }^{2}$ Although this number is not a norm, it is common to call it the $\ell_{0}$-norm [11 14, 19, 33, 57.
} 
nodes as cardinality-constrained SOCP, and recasts it as MISOCP. Section 4 presents a heuristic based on ADMM for the formulation as cardinality-constrained SOCP. Section 5 is devoted to discussion on treatment of overlapping members in a ground structure. Section 6 reports the results of numerical experiments. Some conclusions are drawn in Section 7

In our notation, ${ }^{\top}$ denotes the transpose of a vector or a matrix. We use $\mathbf{1}=(1,1, \ldots, 1)^{\top}$ to denote the all-ones vector. For vectors $\boldsymbol{x}=\left(x_{i}\right) \in \mathbb{R}^{n}$ and $\boldsymbol{y}=\left(y_{i}\right) \in \mathbb{R}^{n}$, we write $\boldsymbol{x} \geq \boldsymbol{y}$ if $x_{i} \geq y_{i}$ $(i=1, \ldots, n)$. We use $\|\boldsymbol{x}\|$ to denote the Euclidean norm (or the $\ell_{2}$-norm) of $\boldsymbol{x}$, i.e., $\|\boldsymbol{x}\|=\sqrt{\boldsymbol{x}^{\top} \boldsymbol{x}}$. We denote by $\|\boldsymbol{x}\|_{0}$ the number of nonzero components of $\boldsymbol{x}$, which is the so-called $\ell_{0}$-norm of $\boldsymbol{x}$. For a finite set $T$, let $|T|$ denote the cardinality of $T$, i.e., the number of elements in $T$. If we define $\operatorname{supp}(\boldsymbol{x}) \subseteq\{1, \ldots, n\}$ by $\operatorname{supp}(\boldsymbol{x})=\left\{i \in\{1, \ldots, n\} \mid x_{i} \neq 0\right\}$, then $\|\boldsymbol{x}\|_{0}=|\operatorname{supp}(\boldsymbol{x})|$. Therefore, a constraint on the $\ell_{0}$-norm is also called the cardinality constraint. For a set $S \subseteq \mathbb{R}^{n}$, we denote by $\delta_{S}: \mathbb{R}^{n} \rightarrow \mathbb{R} \cup\{+\infty\}$ the indicator function of $S$, which is defined by

$$
\delta_{S}(\boldsymbol{x})= \begin{cases}0 & \text { if } \boldsymbol{x} \in S, \\ +\infty & \text { if } \boldsymbol{x} \notin S .\end{cases}
$$

For a closed set $S \subseteq \mathbb{R}^{n}$, the projection of a point $\boldsymbol{z} \in \mathbb{R}^{n}$ onto $S$, denoted $\Pi_{S}(\boldsymbol{z}) \in \mathbb{R}^{n}$, is defined by

$$
\Pi_{S}(\boldsymbol{z}) \in S, \quad\left\|\boldsymbol{z}-\Pi_{S}(\boldsymbol{z})\right\|=\min \{\|\boldsymbol{z}-\boldsymbol{x}\| \mid \boldsymbol{x} \in S\} .
$$

If $S$ is closed and convex, then $\Pi_{S}(\boldsymbol{z})$ exists uniquely for any point $\boldsymbol{z} \in \mathbb{R}^{n}$. The $n$-dimensional second-order cone, denoted $\mathcal{L}^{n}$, is defined by

$$
\mathcal{L}^{n}=\left\{\left(s_{0}, \boldsymbol{s}_{1}\right) \in \mathbb{R} \times \mathbb{R}^{n-1} \mid\left\|\boldsymbol{s}_{1}\right\| \leq s_{0}\right\} .
$$

The $n$-dimensional rotated second-order cone, denoted $\mathcal{K}^{n}$, is defined by

$$
\mathcal{K}^{n}=\left\{(\boldsymbol{x}, y, z) \in \mathbb{R}^{n-2} \times \mathbb{R} \times \mathbb{R} \mid \boldsymbol{x}^{\top} \boldsymbol{x} \leq y z, y \geq 0, z \geq 0\right\} .
$$

We have that $(\boldsymbol{x}, y, z) \in \mathcal{K}^{n}$ if and only if $(y+z, y-z, 2 \boldsymbol{x}) \in \mathcal{L}^{n}$. We use $\mathcal{U}(a, b)$ to denote the continuous uniform distribution on the interval $(a, b) \subset \mathbb{R}$.

\section{Fundamentals of alternating direction method of multipliers}

In this section, we briefly outline the alternating direction method of multipliers (ADMM) for solving convex optimization; see [10] for more accounts.

Let $f: \mathbb{R}^{n} \rightarrow \mathbb{R} \cup\{+\infty\}$ and $g: \mathbb{R}^{m} \rightarrow \mathbb{R} \cup\{+\infty\}$ be closed proper convex functions. Consider the following convex optimization problem in variables $\boldsymbol{x} \in \mathbb{R}^{n}$ and $\boldsymbol{z} \in \mathbb{R}^{m}$ :

$$
\begin{array}{ll}
\underset{\boldsymbol{x}, \boldsymbol{z}}{\operatorname{Minimize}} & f(\boldsymbol{x})+g(\boldsymbol{z}) \\
\text { subject to } & A \boldsymbol{x}+B \boldsymbol{z}=\boldsymbol{c} .
\end{array}
$$

Here, $A \in \mathbb{R}^{l \times n}$ and $B \in \mathbb{R}^{l \times m}$ are constant matrices, and $c \in \mathbb{R}^{l}$ is a constant vector.

The augmented Lagrangian of problem (1) is defined as

$$
L_{\rho}(\boldsymbol{x}, \boldsymbol{z}, \boldsymbol{y})=f(\boldsymbol{x})+g(\boldsymbol{z})+\boldsymbol{y}^{\top}(A \boldsymbol{x}+B \boldsymbol{z}-\boldsymbol{c})+\frac{\rho}{2}\|A \boldsymbol{x}+B \boldsymbol{z}-\boldsymbol{c}\|^{2},
$$


where $\rho>0$ is the penalty parameter, and $\boldsymbol{y} \in \mathbb{R}^{l}$ is the Lagrange multiplier (also called the dual variable). At each iteration of ADMM, we update $\boldsymbol{x}^{k}, \boldsymbol{z}^{k}$, and $\boldsymbol{y}^{k}$ as

$$
\begin{aligned}
\boldsymbol{x}^{k+1} & :=\underset{\boldsymbol{x}}{\arg \min } L_{\rho}\left(\boldsymbol{x}, \boldsymbol{z}^{k}, \boldsymbol{y}^{k}\right), \\
\boldsymbol{z}^{k+1} & :=\underset{\boldsymbol{z}}{\arg \min } L_{\rho}\left(\boldsymbol{x}^{k+1}, \boldsymbol{z}, \boldsymbol{y}^{k}\right), \\
\boldsymbol{y}^{k+1} & :=\boldsymbol{y}^{k}+\rho\left(A \boldsymbol{x}^{k+1}+B \boldsymbol{z}^{k+1}-\boldsymbol{c}\right) .
\end{aligned}
$$

The so-called scaled form of ADMM is defined below. Letting $\boldsymbol{v}=\boldsymbol{y} / \rho$, we see that (2) is reduced to

$$
\tilde{L}_{\rho}(\boldsymbol{x}, \boldsymbol{z}, \boldsymbol{v})=f(\boldsymbol{x})+g(\boldsymbol{z})+\frac{\rho}{2}\|A \boldsymbol{x}+B \boldsymbol{z}-\boldsymbol{c}+\boldsymbol{v}\|^{2}-\frac{\rho}{2}\|\boldsymbol{v}\|^{2} .
$$

By using $\tilde{L}_{\rho}$ in (6), the iteration of ADMM given by (3), (4), and (5) is written as

$$
\begin{aligned}
\boldsymbol{x}^{k+1} & :=\underset{\boldsymbol{x}}{\arg \min } \tilde{L}_{\rho}\left(\boldsymbol{x}, \boldsymbol{z}^{k}, \boldsymbol{v}^{k}\right), \\
\boldsymbol{z}^{k+1} & :=\underset{\boldsymbol{z}}{\arg \min } \tilde{L}_{\rho}\left(\boldsymbol{x}^{k+1}, \boldsymbol{z}, \boldsymbol{v}^{k}\right), \\
\boldsymbol{v}^{k+1} & :=\boldsymbol{v}^{k}+A \boldsymbol{x}^{k+1}+B \boldsymbol{z}^{k+1}-\boldsymbol{c} .
\end{aligned}
$$

The form given in (7), (8), and (9) is called the scaled form of ADMM, and $\boldsymbol{v}$ is called the scaled dual variable.

Primarily, ADMM is an algorithm for solving convex optimization. It is known that ADMM can often serve as an efficient heuristic for diverse nonconvex optimization problems; see, e.g., [15, 16, 26, 36, [53], and [10, Section 9]. For nonconvex problems, ADMM does not necessarily converge. Also, when it converges, the obtained solution is not necessarily optimal. Furthermore, the obtained solution can depend on the penalty parameter and the initial point. Nevertheless, ADMM can be a simple algorithm, and can be efficient in the sense that it often converges to a solution with a good objective value.

\section{Design optimization with limited number of nodes}

In Section 3.1. we define truss topology optimization under the upper bound constraint on the number of nodes. In Section 3.2, we show that this problem can be recast as MISOCP.

\subsection{Problem setting}

Following the ground structure approach, consider an initial truss consisting of many candidate members that are connected by nodes with the given locations. The cross-sectional areas of the members are treated as the design variables to be optimized. It is worth noting that the ground structure may involve some overlapping members, as an example shown in Figure 1. The necessity, as well as the treatment, of overlapping members in a ground structure is thoroughly discussed in Section 5. We use $m, l$, and $d$ to denote the number of members, the number of nodes, and the number of degrees of freedom of the nodal displacements, respectively. 


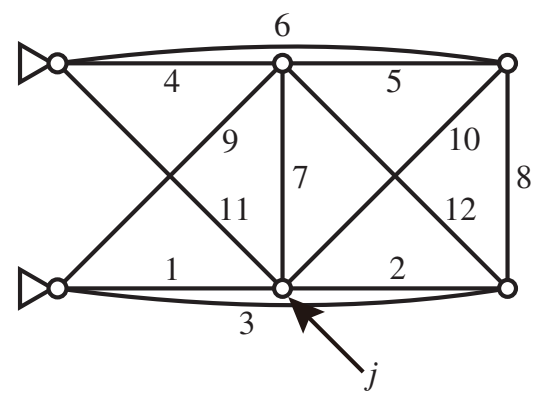

Figure 1: An example of ground structure with overlapping members.

Let $x_{i}(i=1, \ldots, m)$ denote the member cross-sectional areas. We use $K(\boldsymbol{x}) \in \mathbb{R}^{d \times d}$ to denote the stiffness matrix, which can be written as

$$
K(\boldsymbol{x})=\sum_{i=1}^{m} \frac{E}{c_{i}} x_{i} \boldsymbol{b}_{i} \boldsymbol{b}_{i}^{\top} .
$$

Here, $c_{i}$ is the undeformed member length, $E$ is the Young modulus, and $\boldsymbol{b}_{i} \in \mathbb{R}^{d}$ is a constant vector reflecting the member connectivity and the direction cosine of member $i$. For a given external load vector $\boldsymbol{p} \in \mathbb{R}^{d}$, the compliance of the truss, denoted $\pi(\boldsymbol{x})$, is defined by

$$
\pi(\boldsymbol{x})=\sup \left\{2 \boldsymbol{p}^{\top} \boldsymbol{u}-\boldsymbol{u}^{\top} K(\boldsymbol{x}) \boldsymbol{u} \mid \boldsymbol{u} \in \mathbb{R}^{d}\right\} .
$$

Let $V(>0)$ denote the specified upper bound for the structural volume. The conventional compliance minimization problem is formulated as follows:

$$
\begin{array}{cl}
\underset{\boldsymbol{x}}{\operatorname{Minimize}} & \pi(\boldsymbol{x}) \\
\text { subject to } & \boldsymbol{x} \geq \mathbf{0}, \\
& \boldsymbol{c}^{\top} \boldsymbol{x} \leq V .
\end{array}
$$

This problem is convex, and can be recast as follows [7, Section 3.4.3]:

$$
\begin{array}{ll}
\underset{\boldsymbol{x}, \boldsymbol{q}, \boldsymbol{w}}{\operatorname{Minimize}} & \sum_{i=1}^{m} w_{i} \\
\text { subject to } & w_{i} x_{i} \geq \frac{c_{i}}{E} q_{i}^{2}, \quad i=1, \ldots, m, \\
& \boldsymbol{x} \geq \mathbf{0} \\
& \sum_{i=1}^{m} q_{i} \boldsymbol{b}_{i}=\boldsymbol{p}, \\
& \boldsymbol{c}^{\top} \boldsymbol{x} \leq V .
\end{array}
$$

Constraints $12 \mathrm{~b}$ ) and $12 \mathrm{c}$ can be rewritten equivalently as the rotated second-order cone constraints

$$
\left(\sqrt{c_{i} / E} q_{i}, w_{i}, x_{i}\right) \in \mathcal{K}^{3}, \quad i=1, \ldots, m .
$$

These constraints also can be rewritten equivalently as the second-order cone constraints

$$
w_{i}+x_{i} \geq\left\|\left[\begin{array}{c}
w_{i}-x_{i} \\
2 \sqrt{c_{i} / E} q_{i}
\end{array}\right]\right\|, \quad i=1, \ldots, m .
$$


Thus, the conventional compliance minimization, 12, can be recast as SOCP [7, Section 3.4.3]; see also [28, 32].

We are now in position to consider the upper bound constraint on the number of nodes in a truss design. Let $n$ denote the specified upper bound. For the $j$ th node $(j=1, \ldots, l)$, define $I(j) \subseteq\{1, \ldots, m\}$ as the set of indices of the members connected to node $j$. For example, in the case of Figure 1 we have $I(j)=\{1,2,7,10,11\}$. Define $z_{j}(j=1, \ldots, l)$ by

$$
z_{j}=\sum_{i \in I(j)} x_{i}
$$

to see that the number of nodes becomes equal to $\|\boldsymbol{z}\|_{0}$. For notational simplicity, we write 13 as

$$
\boldsymbol{z}=Z \boldsymbol{x}
$$

with a constant matrix $Z \in \mathbb{R}^{l \times m}$. The upshot is that the compliance minimization under the upper bound constraint for the number of existing nodes is formulated as follows:

$$
\begin{array}{cl}
\underset{\boldsymbol{x}, \boldsymbol{z}}{\operatorname{Minimize}} & \pi(\boldsymbol{x}) \\
\text { subject to } & \boldsymbol{x} \geq \mathbf{0}, \\
& \boldsymbol{c}^{\top} \boldsymbol{x} \leq V, \\
& \boldsymbol{z}=Z \boldsymbol{x}, \\
& \|\boldsymbol{z}\|_{0} \leq n .
\end{array}
$$

As mentioned above, the conventional compliance minimization in (11) can be recast as SOCP. Therefore, problem (14) can be reduced to cardinality-constrained SOCP. In Section 3.2, we present its MISOCP reformulation.

\subsection{MISOCP formulation}

In this section, we show that problem (14) can be recast as MISOCP.

For node $j(j=1, \ldots, l)$, we introduce a new variable, $s_{j} \in\{0,1\}$, to indicate whether the node vanishes $\left(s_{j}=0\right)$ or exists $\left(s_{j}=1\right)$. The relation between $s_{j}$ and $z_{j}$ can be given as

$$
0 \leq z_{j} \leq M s_{j}
$$

where $M>0$ is a sufficiently large constant. The upper bound constraint for the number of existing nodes is written in terms of $s_{1}, \ldots, s_{l}$ as

$$
\sum_{j=1}^{l} s_{j} \leq n .
$$

This observation, in conjunction with the SOCP reformulation of problem (11), concludes that 
problem (14) is reduced to the following MISOCP:

$$
\begin{array}{ll}
\underset{\boldsymbol{x}, \boldsymbol{q}, \boldsymbol{w}, \boldsymbol{z}, \boldsymbol{s}}{\operatorname{Minimize}} & \sum_{i=1}^{m} w_{i} \\
\text { subject to } & w_{i}+x_{i} \geq\left\|\left[\begin{array}{c}
w_{i}-x_{i} \\
2 \sqrt{c_{i} / E} q_{i}
\end{array}\right]\right\|, \quad i=1, \ldots, m, \\
& \sum_{i=1}^{m} q_{i} \boldsymbol{b}_{i}=\boldsymbol{p} \\
& \boldsymbol{c}^{\top} \boldsymbol{x} \leq V \\
& \boldsymbol{z}=Z \boldsymbol{x}, \\
& \boldsymbol{z} \leq M \boldsymbol{s}, \\
& \sum_{j=1}^{l} s_{j} \leq n, \\
& \boldsymbol{s} \in\{0,1\}^{l} .
\end{array}
$$

Here, optimization variables are $\boldsymbol{x} \in \mathbb{R}^{m}, \boldsymbol{q} \in \mathbb{R}^{m}, \boldsymbol{w} \in \mathbb{R}^{m}, \boldsymbol{z} \in \mathbb{R}^{l}$, and $\boldsymbol{s} \in \mathbb{R}^{l}$. Although problem (15) is a fairly straightforward extension of the existing SOCP formulation for problem (11), it cannot be found in literature to the best of the authors' knowledge.

\section{Simple heuristic based on alternating direction method of mul- tipliers}

In this section, we present an ADMM as a heuristic for problem (14).

For notational simplicity, define $F \subseteq \mathbb{R}^{m}$ and $G \subseteq \mathbb{R}^{l}$ by

$$
\begin{aligned}
& F=\left\{\boldsymbol{x} \in \mathbb{R}^{m} \mid \boldsymbol{x} \geq \mathbf{0}, \boldsymbol{c}^{\top} \boldsymbol{x} \leq V\right\}, \\
& G=\left\{\boldsymbol{z} \in \mathbb{R}^{l} \mid\|\boldsymbol{z}\|_{0} \leq n\right\} .
\end{aligned}
$$

We see that problem (14) can be written as follows:

$$
\begin{array}{ll}
\text { Minimize } & \pi(\boldsymbol{x})+\delta_{F}(\boldsymbol{x})+\delta_{G}(\boldsymbol{z}) \\
\text { subject to } & Z \boldsymbol{x}-\boldsymbol{z}=\mathbf{0} .
\end{array}
$$

The augmented Lagrangian for problem $(16)$ is formulated as

$$
L_{\rho}(\boldsymbol{x}, \boldsymbol{z}, \boldsymbol{y})=\pi(\boldsymbol{x})+\delta_{F}(\boldsymbol{x})+\delta_{G}(\boldsymbol{z})+\boldsymbol{y}^{\top}(Z \boldsymbol{x}-\boldsymbol{z})+\frac{\rho}{2}\|Z \boldsymbol{x}-\boldsymbol{z}\|^{2},
$$

where $\rho>0$ is the penalty parameter, and $\boldsymbol{y} \in \mathbb{R}^{l}$ is the Lagrange multiplier. Let $\boldsymbol{v}=\boldsymbol{y} / \rho$ to see that 17 is reduced to

$$
\tilde{L}_{\rho}(\boldsymbol{x}, \boldsymbol{z}, \boldsymbol{v})=\pi(\boldsymbol{x})+\delta_{F}(\boldsymbol{x})+\delta_{G}(\boldsymbol{z})+\frac{\rho}{2}\|Z \boldsymbol{x}-\boldsymbol{z}+\boldsymbol{v}\|^{2}-\frac{\rho}{2}\|\boldsymbol{v}\|^{2} .
$$


Using $\tilde{L}_{\rho}$, we can write the iterations of ADMM in the scaled form as

$$
\begin{aligned}
\boldsymbol{x}^{k+1} & :=\underset{\boldsymbol{x}}{\arg \min }\left\{\pi(\boldsymbol{x})+\delta_{F}(\boldsymbol{x})+\frac{\rho}{2}\left\|Z \boldsymbol{x}-\boldsymbol{z}^{k}+\boldsymbol{v}^{k}\right\|^{2}\right\}, \\
\boldsymbol{z}^{k+1} & :=\underset{\boldsymbol{z}}{\arg \min }\left\{\delta_{G}(\boldsymbol{z})+\frac{\rho}{2}\left\|Z \boldsymbol{x}^{k+1}-\boldsymbol{z}+\boldsymbol{v}^{k}\right\|^{2}\right\}, \\
\boldsymbol{v}^{k+1} & :=\boldsymbol{v}^{k}+Z \boldsymbol{x}^{k+1}-\boldsymbol{z}^{k+1} .
\end{aligned}
$$

The first step of ADMM in (18) means that we let $\boldsymbol{x}^{k+1}$ be an optimal solution of the following convex optimization problem:

$$
\begin{array}{ll}
\text { Minimize } & \pi(\boldsymbol{x})+\frac{\rho}{2}\left\|Z \boldsymbol{x}-\boldsymbol{z}^{k}+\boldsymbol{v}^{k}\right\|^{2} \\
\text { subject to } & \boldsymbol{x} \geq \mathbf{0} \\
& \boldsymbol{c}^{\top} \boldsymbol{x} \leq V .
\end{array}
$$

This problem can be recast as SOCP. To see this, using the SOCP formulation of problem (11), we rewrite problem (21) as follows:

$$
\begin{array}{ll}
\text { Minimize } & \sum_{i=1}^{m} w_{i}+\frac{\rho}{2} t \\
\text { subject to } & t \geq\left\|Z \boldsymbol{x}-\boldsymbol{z}^{k}+\boldsymbol{v}^{k}\right\|^{2}, \\
& w_{i}+x_{i} \geq\left\|\left[\begin{array}{c}
w_{i}-x_{i} \\
2 \sqrt{c_{i} / E} q_{i}
\end{array}\right]\right\|, \quad i=1, \ldots, m, \\
& \sum_{i=1}^{m} q_{i} \boldsymbol{b}_{i}=\boldsymbol{p}, \\
& \boldsymbol{c}^{\top} \boldsymbol{x} \leq V,
\end{array}
$$

where $t \in \mathbb{R}$ is an auxiliary variable. Since constraint $(22 \mathrm{~b})$ is a rotated second-order cone constraint $t^{3}$

$$
\left(Z \boldsymbol{x}-\boldsymbol{z}^{k}+\boldsymbol{v}^{k}, t, 1\right) \in \mathcal{K}^{l+2},
$$

problem 22] is an SOCP. We adopt a primal-dual interior-point method for solving this problem. Next, the second step of ADMM in (19) can be written as

$$
\boldsymbol{z}^{k+1} \in \Pi_{G}\left(Z \boldsymbol{x}^{k+1}+\boldsymbol{v}^{k}\right),
$$

where $\Pi_{G}$ is the projection onto $G !^{4}$ We can compute (23) easily [10, Chap. 9]; for a point $z \in \mathbb{R}^{l}$, $\Pi_{G}(\boldsymbol{z})$ keeps the $n$ largest magnitude components of $\boldsymbol{z}$ and zeros out the other components. In this way, each step of ADMM in (18), (19), and (20) can be carried out very easily.

${ }^{3}$ It can also be rewritten as

$$
t+1 \geq\left\|\left[\begin{array}{c}
t-1 \\
2\left(Z \boldsymbol{x}-\boldsymbol{z}^{k}+\boldsymbol{v}^{k}\right)
\end{array}\right]\right\|,
$$

which is a second-order cone constraint.

${ }^{4}$ Since $G$ is nonconvex, the projection of a point onto $G$ is not necessarily unique. 


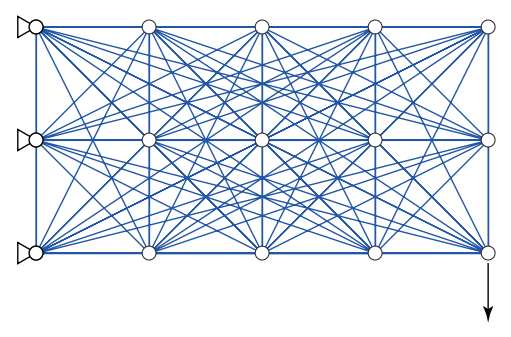

(a)

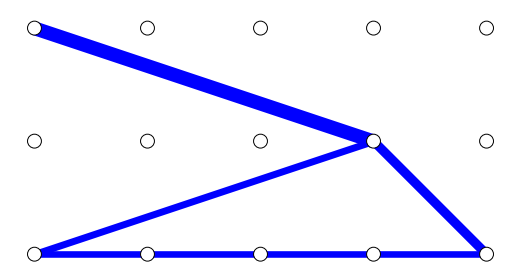

(b)

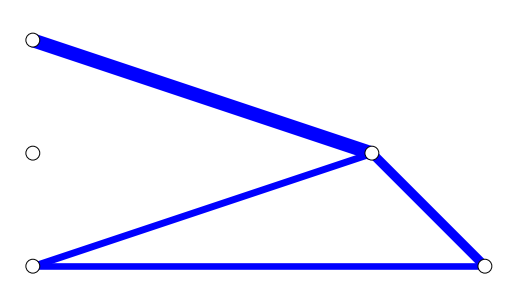

(c)

Figure 2: An example of truss topology optimization and hinge cancellation. (a) The problem setting; (b) the optimal solution; and (c) the final design after hinge cancellation.

\section{On overlapping members}

Unlike the conventional compliance minimization of a truss, overlapping members in a ground structure are not redundant for the optimization problem considered in this paper. This section explains the treatment of overlapping members.

We begin by reviewing that overlapping members in a ground structure is redundant for the conventional compliance minimization of a truss. For example, consider the ground structure shown in Figure 2(a), Here, any two nodes are connected by a member, but overlapping of members is avoided by removing the longer member when two members overlap. The leftmost nodes are pinsupported. The vertical external force is applied to the bottom rightmost node. Figure 2(b) shows the optimal solution of the compliance minimization, i.e., problem (11). This solution has four horizontal consecutive members that are connected by nodes supported only in the direction of those members. A sequence of such members is sometimes called a chain [1]. In this example, without changing the objective value, we can remove three intermediate nodes to replace the chain with a single longer member. This procedure is called the hinge cancellation [1, 50]. As a result of hinge cancellation, we obtain the final truss design shown in Figure 2(c), Thus, longer overlapping members, like the horizontal member in Figure 2(c), are unnecessary to a ground structure. In contrast, when we consider a constraint on the number of nodes, the optimal solution depends on existence of overlapping members in a ground structure. For example, the truss in Figure 2(b) has five free nodes, while the one in Figure 2(c) has two free nodes. Thus, the hinge cancellation can possibly change the feasibility of the cardinality constraint and, hence, overlapping members in a ground structure are not redundant ${ }^{5}$

\footnotetext{
${ }^{5}$ Such non-redundancy of overlapping members is also known for truss topology optimization considering, e.g., the self-weight load [8, 30] and the member buckling constraints [24, 40].
} 
When we consider a ground structure with some overlapping members, existence of overlapping members in an obtained solution is not allowed from a practical point of view. The method proposed in Section 4 does not consider explicitly the constraint prohibiting presence of overlapping members. Nevertheless, in practice, a solution obtained by the proposed method often has no overlapping members, as illustrated through numerical experiments in Section 4.

Within the framework of MISOCP, we can explicitly incorporate the constraints prohibiting the presence of mutually overlapping members in a truss design. To do this, besides $s \in\{0,1\}^{l}$ in Section 3.2 , we use extra $0-1$ variables $\boldsymbol{t} \in\{0,1\}^{m}$ to indicate whether each member vanishes or exists. Namely, $t_{i}=0$ means that member $i$ is removed, while $t_{i}=1$ means that member $i$ exists. The relation between $t_{i}$ and $x_{i}$ is given by

$$
0 \leq x_{i} \leq M t_{i}
$$

where $M>0$ is a sufficiently large constant. Recall that $I(j)$ denotes the set of indices of the members connected to node $j$; see Section 4 . The relation between $t_{i}(i \in I(j))$ and $s_{j}$ is given by

$$
t_{i} \leq s_{j}, \quad \forall i \in I(j) .
$$

Let $D$ denote the set of pairs of indices of the members that mutually overlap. Namely, $\left(i_{1}, i_{2}\right) \in D$ means that member $i_{1}$ and member $i_{2}$ cannot exist simultaneously. This constraint is written as

$$
t_{i_{1}}+t_{i_{2}} \leq 1, \quad \forall\left(i_{1}, i_{2}\right) \in D
$$

The upshot is that the truss topology optimization problem can be formulated as the following MISOCP:

$$
\begin{array}{ll}
\text { Minimize } & \sum_{i=1}^{m} w_{i} \\
\text { subject to } & w_{i}+x_{i} \geq\left\|\left[\begin{array}{c}
w_{i}-x_{i} \\
2 \sqrt{c_{i} / E} q_{i}
\end{array}\right]\right\|, \quad i=1, \ldots, m, \\
& \sum_{i=1}^{m} q_{i} \boldsymbol{b}_{i}=\boldsymbol{p}, \\
& \boldsymbol{c}^{\top} \boldsymbol{x} \leq V, \\
& \boldsymbol{x} \leq M \boldsymbol{t}, \\
& t_{i} \leq s_{j}(\forall i \in I(j)), \quad j=1, \ldots, l, \\
& \boldsymbol{s} \geq \mathbf{0}, \\
& \sum_{j=1}^{l} s_{j} \leq n, \\
& t_{i_{1}}+t_{i_{2}} \leq 1, \quad \forall\left(i_{1}, i_{2}\right) \in D, \\
& \boldsymbol{t} \in\{0,1\}^{m} .
\end{array}
$$

It is worth noting that the $0-1$ constraints on $s_{1}, \ldots, s_{k}$ can be omitted. 


\section{$6 \quad$ Numerical experiments}

In this section, we report numerical experiments on the method presented in Section 4. In Section 6.1. we describe the details of implementation of the algorithm and the problem settings of the numerical experiments. The computational results of the proposed ADMM approach, together with the comparison with the MISOCP approach, are presented in Sections 6.2, 6.3, and 6.4. Empirical evidences of our stopping criterion and selection of initial points are presented in Sections 6.5 and 6.6. respectively. Section 6.7 presents application of the proposed method to robust truss optimization, which is recast as mixed-integer semidefinite programming.

\subsection{Implementation and problem settings}

At each iteration of the proposed method, we solved problem (22) by using CVX ver. 2.1, a MATLAB package for specifying and solving convex optimization problems [20, 21]. As a solver, we used SDPT3 ver. 4.0 [56] on MATLAB ver. 9.1.0. The cvx_precision of CVX is set to best, which means that the solver continues as far as it can make progress [21. For comparison, we solved the MISOCP problem in (15) with a global optimization approach. The value of $M$ in constraint (15f) is set to $1.0 \times 10^{5}$ in $\mathrm{m}^{6}$ We used PICOS ver. 1.1.2, a Python interface to diverse optimization solvers [51]. MOSEK ver. 8.0.1 [2] was used as the solver. Computation was carried out on two 3.2 GHz Intel Xeon E5-2667 v4 processors with 256 GB RAM.

In practice, we slightly modify the original version of ADMM introduced in Section 2 so that the penalty parameter in the augmented Lagrangian is gradually increased. Specifically, $\rho$ in subproblem 22 is given by

$$
\rho_{k+1}:=\min \left\{\mu \rho_{k}, \rho_{\max }\right\}
$$

where $\mu(>1)$ and $\rho_{\max }\left(>\rho_{0}\right)$ are constants. In the following, we set $\mu=1.5, \rho_{0}=1$, and $\rho_{\text {max }}=10^{6}$. Define $J_{0}^{k} \subseteq\{1, \ldots, l\}$ by

$$
J_{0}^{k}=\left\{j \in\{1, \ldots, l\} \mid z_{j}^{k} \leq \epsilon\right\},
$$

where we set $\epsilon=0.1 \mathrm{~mm}^{2}$. We terminate the ADMM when

$$
l-\left|J_{0}^{k}\right| \leq n
$$

is satisfied. Then we solve problem (11) with the additional constraints

$$
\sum_{i \in I(j)} x_{i}=0, \quad \forall j \in J_{0}^{k}
$$

to generate the final output. As for the initial point for the ADMM, we examine two cases:

- Initial point $(\mathrm{A}): \boldsymbol{z}^{0}:=Z \boldsymbol{x}^{0}$ and $\boldsymbol{v}^{0}:=\mathbf{0}$, where $\boldsymbol{x}^{0}$ is an optimal solution of problem (11).

- Initial point $(\mathrm{B}): \boldsymbol{z}^{0}:=Z \boldsymbol{x}^{0}$ and $\boldsymbol{v}^{0}:=\mathbf{0}$ with $\boldsymbol{x}^{(0)}:=\left(V / \boldsymbol{c}^{\top} \mathbf{1}\right) \mathbf{1}$.

\footnotetext{
${ }^{6}$ Through our preliminary numerical experiments it was found that the computational cost required by MOSEK does not change drastically depending on the value of $M$.
} 
It should be clear that only $\boldsymbol{z}^{0}$ and $\boldsymbol{v}^{0}$ are used as input data of the ADMM; $\boldsymbol{x}^{0}$ is not required as input.

Consider the problem setting shown in Figure 3 . The nodes are aligned on a $1 \mathrm{~m} \times 1 \mathrm{~m}$ grid. We vary the values of $N_{X}$ and $N_{Y}$ to generate problem instances with diverse sizes. The number of free nodes in this ground structure is $N_{X}\left(N_{Y}+1\right)$. The members in a ground structure are generated as follows: We first consider all possible members such that any two nodes are connected by a member. Then we remove members that are longer than a specified value, $5 \mathrm{~m}$ in Sections 6.2 and 6.3 while $7 \mathrm{~m}$ in Section 6.4. It is worth noting that the ground structure retains overlapping members.

In the following examples, the Young modulus is $E=20 \mathrm{GPa}$, and the specified upper bound for the structural volume is $V=2 N_{X} N_{Y} \times 10^{5} \mathrm{~mm}^{3}$. As for $\boldsymbol{p}$, the external vertical force of $100 \mathrm{kN}$ is applied to the bottom rightmost node. We consider $n$ as the upper bound for the number of free nodes. In other words, the number of supports is not restricted in the following examples, and $l$ in the previous sections denotes the number of free nodes of a ground structure.

Table 1: Characteristics of the problem instances for the numerical experiments.

\begin{tabular}{lrrrr}
\hline$\left(N_{X}, N_{Y}\right)$ & $m$ & $d$ & $\hat{w}(\mathrm{~J})$ & \#free nodes \\
\hline$(5,2)$ & 147 & 30 & 12100.00 & 9 \\
$(5,3)$ & 264 & 40 & 5007.41 & 7 \\
$(5,4)$ & 411 & 50 & 2812.50 & 5 \\
\hline$(8,2)$ & 273 & 48 & 34515.63 & 15 \\
$(9,2)$ & 315 & 54 & 45125.00 & 8 \\
$(8,4)$ & 750 & 80 & 6937.81 & 10 \\
$(9,4)$ & 863 & 90 & 8900.28 & 10 \\
$(8,6)$ & 1296 & 112 & 3080.39 & 10 \\
$(9,6)$ & 1489 & 126 & 3847.25 & 12 \\
\hline
\end{tabular}

Table 2: Computational results of example (I).

\begin{tabular}{|c|c|c|c|c|c|c|c|}
\hline \multirow[b]{2}{*}{$\left(N_{X}, N_{Y}\right)$} & \multicolumn{5}{|c|}{ ADMM } & \multicolumn{2}{|c|}{ MISOCP } \\
\hline & Init. sol. & $w^{*}(\mathrm{~J})$ & $w^{*} / \hat{w}$ & \#iter & Time (s) & $\bar{w}(\mathrm{~J})$ & Time (s) \\
\hline \multirow[t]{2}{*}{$(5,2)$} & $*(\mathrm{~A})$ & 12100.00 & 1.000 & 5 & 3.6 & 12100.00 & 0.72 \\
\hline & $*(\mathrm{~B})$ & 12100.00 & 1.000 & 3 & 2.2 & & \\
\hline \multirow[t]{2}{*}{$(5,3)$} & $*(\mathrm{~A})$ & 5007.41 & 1.000 & 5 & 3.2 & 5007.41 & 1.69 \\
\hline & (B) & 5052.45 & - & 3 & 2.0 & & \\
\hline \multirow[t]{2}{*}{$(5,4)$} & $*(\mathrm{~A})$ & 2812.50 & 1.000 & 2 & 2.6 & 2812.50 & 0.52 \\
\hline & $*(\mathrm{~B})$ & 2812.50 & 1.000 & 3 & 3.8 & & \\
\hline
\end{tabular}




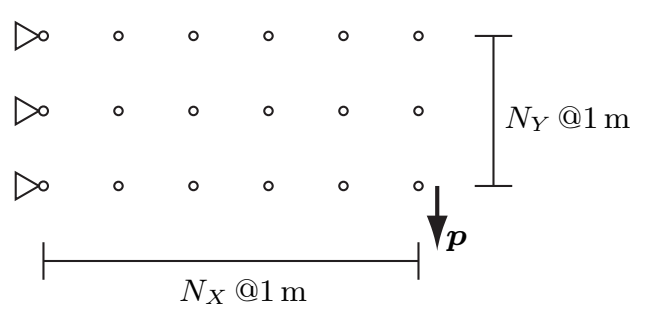

Figure 3: The problem setting for numerical experiments with $\left(N_{X}, N_{Y}\right)=(5,2)$.

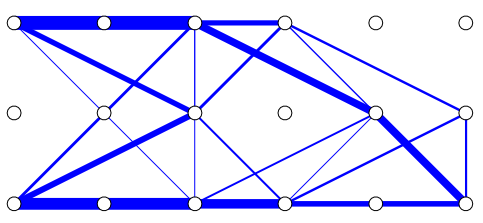

(a)

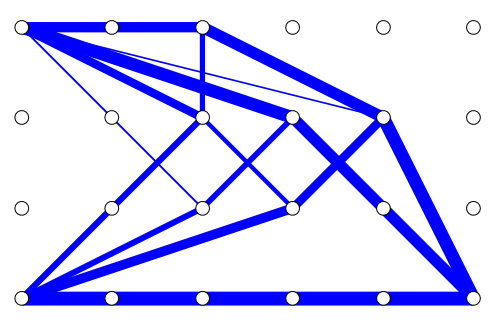

(b)

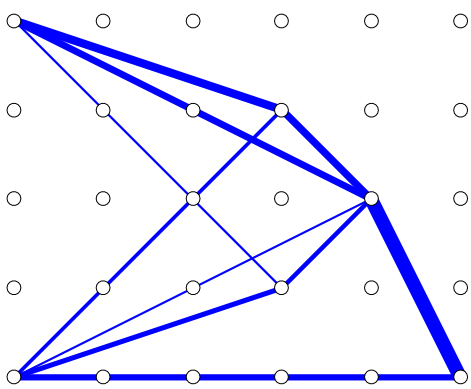

(c)

Figure 4: Example (I). The optimal solutions of the compliance minimization (without the cardinality constraint). (a) $\left(N_{X}, N_{Y}\right)=(5,2)$; (b) $\left(N_{X}, N_{Y}\right)=(5,3)$; and (c) $\left(N_{X}, N_{Y}\right)=(5,4)$.

\subsection{Example (I)}

In this section, we set the upper bound for the existing free nodes to $n=4$. As for problem instances, consider $\left(N_{X}, N_{Y}\right)=(5,2),(5,3)$, and $(5,4)$ in Figure 3 . Figure 4 shows the optimal solutions 7 of the conventional compliance minimization without the constraint on the number of nodes, i.e., problem (11), where the width of each member is proportional to its cross-sectional area. Table 1 reports the optimal values, denoted $\hat{w}$. It also lists the number of members $(m)$ and the number of degrees of freedom of the nodal displacements $(d)$. As mentioned in Section 5 , the intermediate nodes on a chain in Figure 4 can be removed without changing the objective value. After this hinge cancellation procedure, the numbers of free nodes in Figures (a) (b), and (c) become 9, 7, and 5, respectively, as listed in Table 1 .

Figure 5 shows the solutions obtained by the proposed ADMM for the problem with the limited number of free nodes. In Figure 5(b) we see that the number of free nodes is $3(<n)$. It should be clear that $\boldsymbol{x}^{0}$ used to generate initial point (A) for the ADMM is in general different from the one in Figure 4 , because $\boldsymbol{x}^{0}$ is computed from the ground structure involving the overlapping members. Indeed, $\boldsymbol{x}^{0}$ for $\left(N_{X}, N_{Y}\right)=(5,2),(5,3)$, and $(5,4)$ have 12,15 , and 13 free nodes, respectively.

\footnotetext{
7 To obtain these solutions, we used the ground structures without overlapping members.
} 


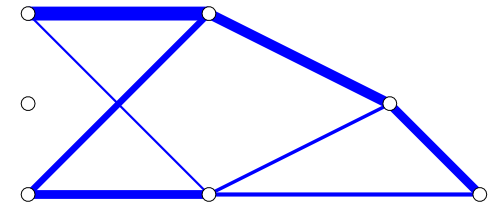

(a)

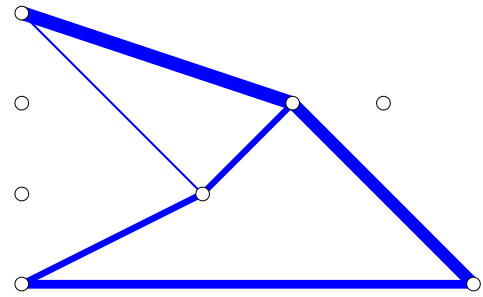

(b)

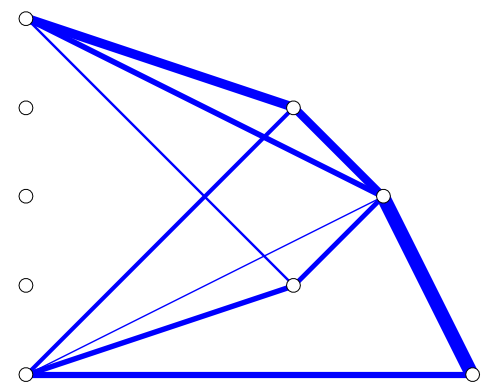

(c)

Figure 5: Example (I). The solutions obtained by the proposed method for the compliance minimization with the cardinality constraint $(n=4)$. (a) $\left(N_{X}, N_{Y}\right)=(5,2) ;(\mathrm{b})\left(N_{X}, N_{Y}\right)=(5,3)$; and (c) $\left(N_{X}, N_{Y}\right)=(5,4)$.

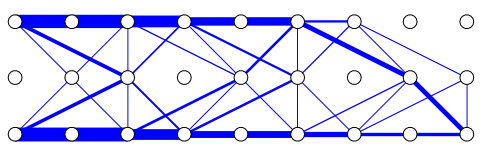

(a)

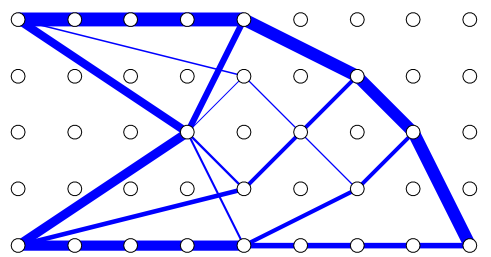

(c)

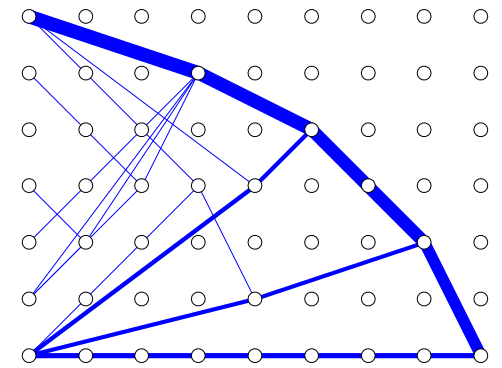

(e)

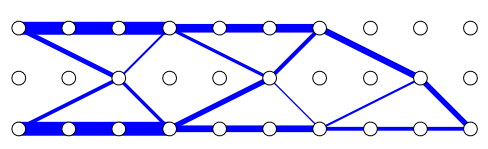

(b)

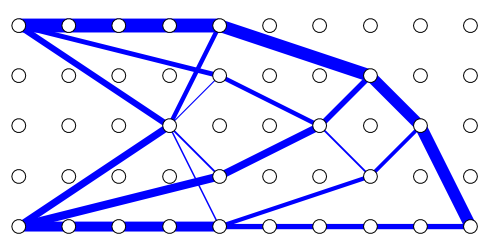

(d)

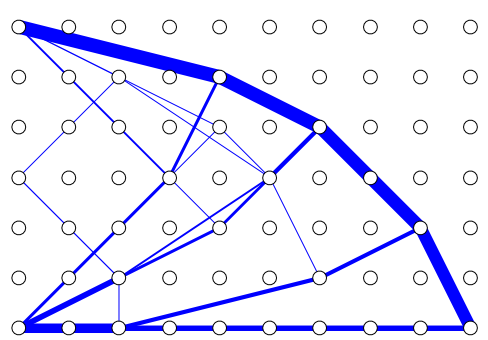

(f)

Figure 6: Example (II). The optimal solutions of the compliance minimization (without the cardinality constraint). (a) $\left(N_{X}, N_{Y}\right)=(8,2)$; (b) $\left(N_{X}, N_{Y}\right)=(9,2) ;(\mathrm{c})\left(N_{X}, N_{Y}\right)=(8,4)$; (d) $\left(N_{X}, N_{Y}\right)=(9,4) ;(\mathrm{e})\left(N_{X}, N_{Y}\right)=(8,6) ;$ and $(\mathrm{f})\left(N_{X}, N_{Y}\right)=(9,6)$. 


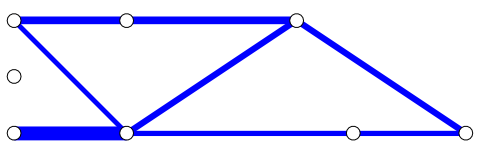

(a)

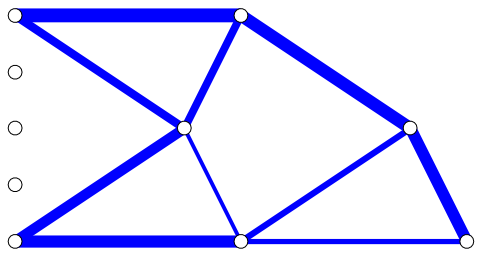

(c)

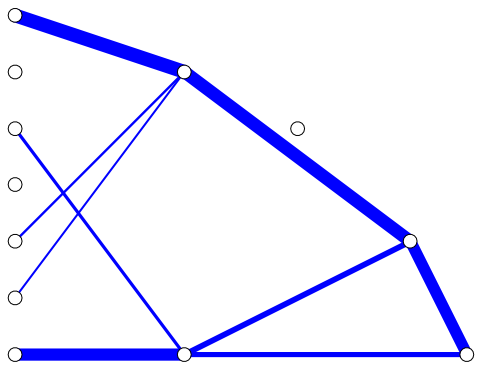

(e)

Figure 7: Example (II). The solutions obtained by the ADMM for the compliance minimization with the cardinality constraint $(n=5)$. (a) $\left(N_{X}, N_{Y}\right)=(8,2)$; (b) $\left(N_{X}, N_{Y}\right)=(9,2)$; (c) $\left(N_{X}, N_{Y}\right)=$ $(8,4) ;(\mathrm{d})\left(N_{X}, N_{Y}\right)=(9,4) ;(\mathrm{e})\left(N_{X}, N_{Y}\right)=(8,6) ;$ and $(\mathrm{f})\left(N_{X}, N_{Y}\right)=(9,6)$.

Thus, the number of nodes is decreased successfully by the proposed method. It is observed that the solutions in Figure 4(a) and Figure 4(b) have too many members from a practical point of view. In contrast, we can see in Figure 5(a) and Figure 5(b) that the number of members is decreased as a result of optimization with the limitation of the number of nodes. The computational results of the ADMM are listed in Table 2, where $w^{*}$ is the objective value of the obtained solution, "\#iter." is the number of iterations required before convergence, and "time" is the computational time. As mentioned before, we examine two different values, denoted (A) and (B), for $\boldsymbol{z}^{0}$ and $\boldsymbol{v}^{0}$. The one which yields the better objective value is indicated by "*." It is observed in Table 2 that, for every instance, the objective value of the solution obtained by the ADMM approach is identical to the optimal value of the problem without the limitation of the number of nodes (i.e., problem (11)). Since problem (11) can be regarded as a relaxation problem, the solutions obtained by the proposed ADMM are globally optimal. This also illustrates that, in general, the compliance minimization of a truss has more than one optimal solution, and the optimal solutions may have different numbers of nodes.

For comparison, we also solved MISOCP (15) with a global optimization approach. Table 2 lists the obtained results 8 where $\bar{w}$ is the objective value. The solutions obtained by the MISOCP solver are identical to the ones obtained by the ADMM approach.

\footnotetext{
${ }^{8}$ It should be clear that no initial point was assigned for the MISOCP approach, although in Table 2, for convenience of presentation, the results of MISOCP are placed in the rows concerning the results of the ADMM with initial point (A).
} 


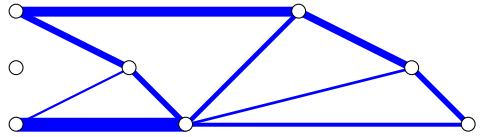

(a)

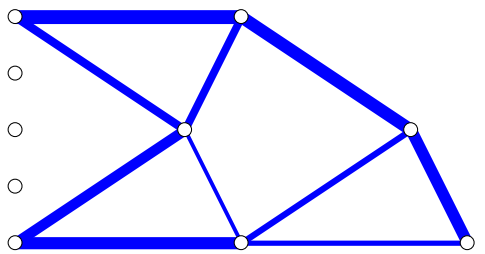

(c)

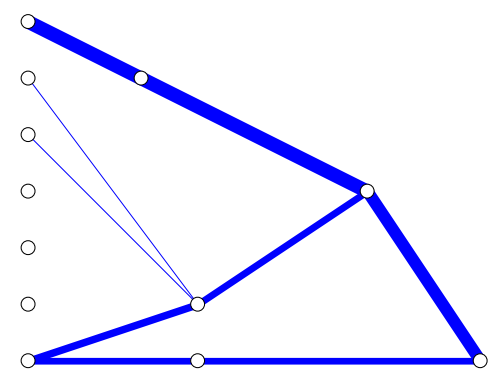

(e)

Figure 8: Example (II). The optimal solutions obtained by MISOCP for the compliance minimization with the cardinality constraint $(n=5)$. (a) $\left(N_{X}, N_{Y}\right)=(8,2) ;(\mathrm{b})\left(N_{X}, N_{Y}\right)=(9,2)$; (c) $\left(N_{X}, N_{Y}\right)=(8,4) ;(\mathrm{d})\left(N_{X}, N_{Y}\right)=(9,4) ;(\mathrm{e})\left(N_{X}, N_{Y}\right)=(8,6)$; and $(\mathrm{f})\left(N_{X}, N_{Y}\right)=(9,6)$.

Table 3: Computational results of example (II).

\begin{tabular}{|c|c|c|c|c|c|c|c|c|}
\hline \multirow[b]{2}{*}{$\left(N_{X}, N_{Y}\right)$} & \multicolumn{6}{|c|}{$\mathrm{ADMM}$} & \multicolumn{2}{|c|}{ MISOCP } \\
\hline & Init. sol. & $w^{*}(\mathrm{~J})$ & $w^{*} / \hat{w}$ & $w^{*} / \bar{w}$ & \#iter & Time (s) & $\bar{w}(\mathrm{~J})$ & Time (s) \\
\hline \multirow[t]{2}{*}{$(8,2)$} & $*(\mathrm{~A})$ & 37515.63 & 1.087 & 1.072 & 11 & 9.0 & 35006.93 & 13.42 \\
\hline & $*(\mathrm{~B})$ & 37515.63 & 1.087 & 1.072 & 13 & 9.8 & & \\
\hline \multirow[t]{2}{*}{$(9,2)$} & $*(\mathrm{~A})$ & 50000.00 & 1.108 & 1.070 & 20 & 18.8 & 46722.20 & 19.92 \\
\hline & $*(\mathrm{~B})$ & 50000.00 & 1.108 & 1.070 & 18 & 16.5 & & \\
\hline \multirow[t]{2}{*}{$(8,4)$} & $*(\mathrm{~A})$ & 7031.25 & 1.013 & 1.000 & 11 & 46.4 & 7031.25 & 4.30 \\
\hline & $*(\mathrm{~B})$ & 7031.25 & 1.013 & 1.000 & 6 & 24.5 & & \\
\hline \multirow[t]{2}{*}{$(9,4)$} & (A) & 9167.44 & - & - & 13 & 77.2 & & \\
\hline & $*(\mathrm{~B})$ & 9000.00 & 1.011 & 1.000 & 8 & 46.7 & 8999.91 & 12.20 \\
\hline \multirow[t]{2}{*}{$(8,6)$} & $*(\mathrm{~A})$ & 3287.84 & 1.067 & 1.038 & 10 & 80.3 & 3168.98 & 76.22 \\
\hline & (B) & 3440.05 & - & - & 7 & 54.1 & & \\
\hline \multirow[t]{2}{*}{$(9,6)$} & (A) & 4353.91 & - & - & 9 & 96.5 & & \\
\hline & $*(\mathrm{~B})$ & 4221.65 & 1.097 & 1.060 & 12 & 122.1 & 3983.34 & 112.08 \\
\hline
\end{tabular}




\subsection{Example (II)}

As for instances with larger sizes, consider $\left(N_{X}, N_{Y}\right)=(8,2),(9,2),(8,4),(9,4),(8,6)$, and $(9,6)$. In this section, we set the upper bound for the number of free nodes to $n=5$.

\subsubsection{Results}

Figure 6 collects the optimal solutions without limiting the number of nodes. The number of free nodes after applying the hinge cancellation is reported in Table 1 . Figure 7 shows the solutions obtained by the ADMM approach. The number of free nodes in Figure $7(\mathrm{e})$ is $4(<n)$. Two nodes can be removed from the solution in Figure 7(a), which results in a truss design with three free nodes. It is observed in Figure 6 and Figure 7 that the limitation of the number of nodes often yields a solution with a fewer members. Also, too thin members are observed in Figure 6, while such thin members do not appear in Figure 7. These two features of the solutions in Figure 7 are considered practically preferable. The initial design $\boldsymbol{x}^{0}$ used for generating initial points (A) for the ADMM to solve $\left(N_{X}, N_{Y}\right)=(8,2),(9,2), \ldots,(9,6)$ have $20,18,19,20,24$, and 26 free nodes, respectively.

It is observed in Table 3 that the ADMM terminates after at most 20 iterations. Increase of the objective value from the optimal value of the problem without the cardinality constraint is quite small, i.e., increase by at most about $10 \%$. Particularly, for $\left(N_{X}, N_{Y}\right)=(8,4)$ and $(9,4)$ we have only about $1 \%$ increase. Thus, it is often that the number of nodes can be reduced at the expense of only small increase of the compliance.

The computational results of the MISOCP approach are listed in Table 3 . Figure 8 collects the obtained solutions. For $\left(N_{X}, N_{Y}\right)=(8,4)$, the solution obtained by MISOCP is identical to the one obtained by the ADMM; i.e., the ADMM found a global optimal solution. For $\left(N_{X}, N_{Y}\right)=(9,4)$, it is observed in Table 3 that the objective values obtained by the two methods are almost same, but the two solutions are slightly different as seen in Figure 7(d) and Figure 8(d). The largest value of $w^{*} / \bar{w}$ is 1.072 in the case of $\left(N_{X}, N_{Y}\right)=(8,2)$. It is also worth noting that, for $\left(N_{X}, N_{Y}\right)=(8,6)$ and $(9,6)$, although the global optimal solutions in Figure 8(e) and Figure 8(f) involve very thin members, the solutions obtained by the ADMM shown in Figure 7(e) and Figure 7(f) do not have such a thin member.

It is observed from Table 3 that the proposed ADMM often converges more quickly than the MISOCP solver; exceptions are $\left(N_{X}, N_{Y}\right)=(8,4)$ and $(9,4)$. The computational time required by the MISOCP solver varies drastically depending on problem instances. In contrast, the number of iterations required by the ADMM is almost independent of problem instances. Since the computational time required for solving the SOCP subproblem of the ADMM depends on the problem size, it is possible to roughly estimate the total computational cost of the ADMM from the problem size. This might be considered one of advantages of ADMM over MISOCP.

As mentioned in Section 5, the proposed method does not incorporate the constraint prohibiting overlapping members. Nevertheless, for the problems with limitation of the number of nodes, all the solutions obtained in Section 6 do not involve overlapping members. 


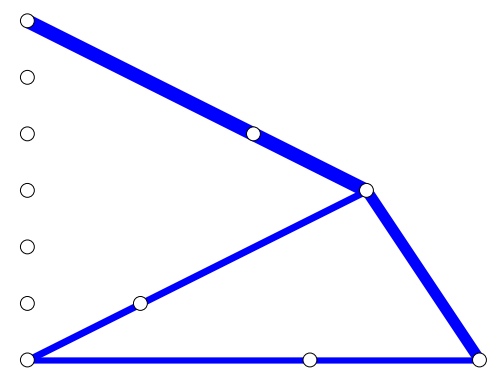

(a)

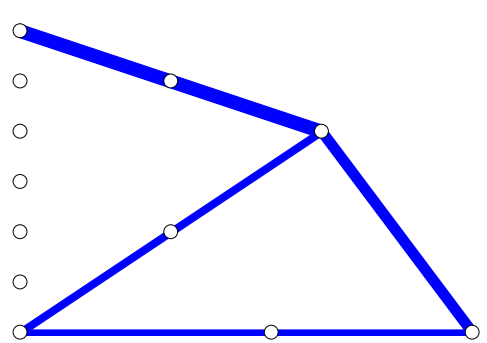

(b)

Figure 9: The optimal solutions of example (II) with the constraints avoiding the presence of thin members. (a) $\left(N_{X}, N_{Y}\right)=(8,6)$; and (b) $\left(N_{X}, N_{Y}\right)=(9,6)$.

\subsubsection{MISOCP with slenderness constraints}

The constraints preventing the presence of very thin members observed in Figure 8(e) and Figure 8(f) can be handled within the framework of mixed-integer programming (MIP) [30. Recall problem (24) in Section 5, where $t_{i}$ is a binary variable indicating whether member $i$ exists or vanishes. Let $x_{\min }>0$ denote the specified lower bound for the member cross-sectional area. The constraint avoiding existence of too thin members can be formulated as

$$
x_{\min } \boldsymbol{t} \leq \boldsymbol{x} \leq M \boldsymbol{t}
$$

In problem (24), we replace constraint (24e) with (25). The constraint avoiding the presence of overlapping members, 24i), is not considered. We solve this MISOCP for the instances $\left(N_{X}, N_{Y}\right)=(8,6)$ and $(9,6)$ with $x_{\min }=200 \mathrm{~mm}^{2}$. The obtained solutions are shown in Figure 9 . Both solutions have parallel consecutive members that are connected by nodes supported only in the direction of those members. The intermediate nodes can be removed without changing the optimal value. Hence, the number of free nodes of these solutions is essentially two. The objective value of the solution for $\left(N_{X}, N_{Y}\right)=(8,6)$ is $3168.97 \mathrm{~J}$, which is slightly less than that for the case without the slenderness constraints in Table 3 . This is due to the computational error in computing the objective value with the finite element method. In the solution shown in Figure 7(e) (i.e., the solution obtained by the ADMM without the slenderness constraints), the cross-sectional area of the thinest member is $75.4 \mathrm{~mm}^{2}$. Hence, this solution is not globally optimal under the slenderness constraint. The computational time required by MOSEK is $365.0 \mathrm{~s}$. The objective value of the solution for $\left(N_{X}, N_{Y}\right)=(9,6)$ is 4028.94J. This is larger than that for the case without the slenderness constraints as expected, and is less than that of the solution obtained by the ADMM. Since the cross-sectional area of the thinnest member of the solution shown in Figure $7(\mathrm{f})$ is $146.7 \mathrm{~mm}^{2}$, Hence, the solution shown in Figure $7(\mathrm{f})$ is not globally optimal under the slenderness constraints. The computational time required by MOSEK to find the solution in Figure 9(b) was 1676.0 s, which is much larger than the computational time of the ADMM.

\subsection{Example (III)}

Consider problem instances $\left(N_{X}, N_{Y}, n\right)=(12,6,6),(13,6,6)$, and $(14,6,7)$. The maximum member length in a ground structure is set to $7 \mathrm{~m}$. 

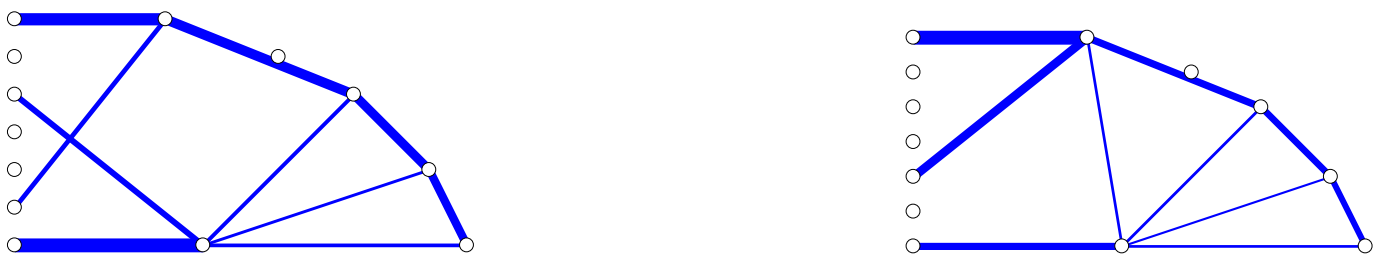

(a)

(b)

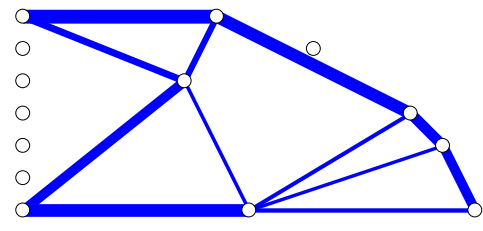

(c)

Figure 10: Example (III). The solutions obtained by ADMM for the compliance minimization with the cardinality constraint. (a) $\left(N_{X}, N_{Y}, n\right)=(12,6,6)$; (b) $\left(N_{X}, N_{Y}, n\right)=(13,6,6)$; and (c) $\left(N_{X}, N_{Y}, n\right)=(14,6,7)$,

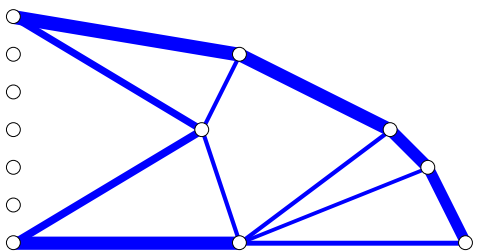

(a)

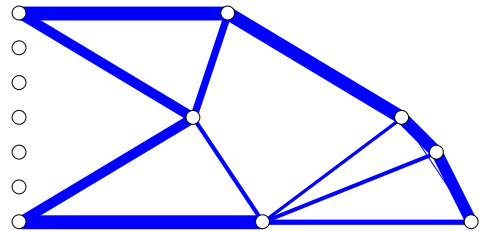

(b)

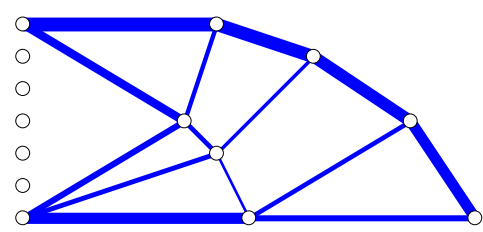

(c)

Figure 11: Example (III). The optimal solutions obtained by MISOCP for the compliance minimization with the cardinality constraint. (a) $\left(N_{X}, N_{Y}, n\right)=(12,6,6)$; (b) $\left(N_{X}, N_{Y}, n\right)=(13,6,6)$; and (c) $\left(N_{X}, N_{Y}, n\right)=(14,6,7)$.

Figure 10 shows the solutions obtained by the proposed ADMM approach. The ADMM terminates with a solution having $n$ free nodes. One of these nodes vanishes in the post-processing. The objective value as well as the computational cost is reported in Table 4 .

Figure 11 collects the optimal solutions found by the MISOCP approach. These solutions use exactly $n$ free nodes. It is observed from Table 4 that the objective value obtained by the ADMM for the largest instance, $\left(N_{X}, N_{Y}, n\right)=(14,6,7)$, is very close to the optimal value. In contrast, for $\left(N_{X}, N_{Y}, n\right)=(13,6,6)$ the objective value obtained by the ADMM is more than $20 \%$ larger than the optimal value. However, the computational cost of the ADMM is much less than the MISOCP approach (which requires more than four hours). Thus, the quality of the solution obtained by the ADMM approach can possibly be very good, although in general it depends on problem instances. As the problem size increases, the computational cost of the ADMM approach becomes much smaller 
Table 4: Computational results of example (III).

\begin{tabular}{lrrrrrrrrr}
\hline & \multicolumn{6}{c}{ ADMM } & & \multicolumn{2}{c}{ MISOCP } \\
\cline { 2 - 3 }$\left(N_{X}, N_{Y}, n\right)$ & Init. sol. & $w^{*}(\mathrm{~J})$ & $w^{*} / \bar{w}$ & $\#$ \#iter & Time $(\mathrm{s})$ & & $\bar{w}(\mathrm{~J})$ & Time $(\mathrm{s})$ \\
\hline$(12,6,6)$ & $*(\mathrm{~A})$ & 7817.72 & 1.115 & 10 & 531.7 & 7012.95 & 3492.34 \\
& $(\mathrm{~B})$ & 9527.47 & & 10 & 516.7 & & \\
\hline$(13,6,6)$ & $*(\mathrm{~A})$ & 10142.88 & 1.228 & 10 & 642.6 & 8258.85 & 14675.17 \\
& $(\mathrm{~B})$ & 13631.69 & & 11 & 793.7 & & \\
\hline$(14,6,7)$ & $*(\mathrm{~A})$ & 9720.09 & 1.018 & 9 & 724.2 & & 9550.97 & 14657.54 \\
& $(\mathrm{~B})$ & 14928.49 & & 14 & 1148.6 & & \\
\hline
\end{tabular}

compared with the MISOCP approach.

\subsection{On heuristic for stopping ADMM}

As mentioned in section 6.1, we use a heuristic criterion for stopping the ADMM. Namely, we stop the ADMM when the cardinality constraint is satisfied with $\epsilon$ tolerance. Then, as for a postprocessing, we solve the compliance minimization problem, (11), with specifying the set of vanishing nodes. This section presents some empirical justification for this procedure. Namely, it is illustrated through numerical experiments that with this heuristic procedure the number of subproblems to be solved is drastically reduced, without missing out better solutions in the sense of the objective valued. We use the problem instances in Section 6.3 .

We performed the following experiment. The ADMM is run until it terminates with a small

Table 5: The computational results when the ADMM is run until the convergence to evaluate effectiveness of the heuristic stopping criterion.

\begin{tabular}{llrrr}
\hline$\left(N_{X}, N_{Y}, n\right)$ & Init. sol. & $\tilde{K}$ & $\tilde{K}_{\mathrm{p}}$ & $K^{*}$ \\
\hline$(8,2,5)$ & $(\mathrm{A})$ & 35 & 18 & 11 \\
$(8,2,5)$ & $(\mathrm{B})$ & 35 & 18 & 13 \\
$(9,2,5)$ & $(\mathrm{A})$ & $100^{\dagger}$ & $77^{\dagger}$ & 20 \\
$(9,2,5)$ & $(\mathrm{B})$ & 81 & 58 & 18 \\
$(8,4,5)$ & $(\mathrm{A})$ & 27 & 15 & 11 \\
$(8,4,5)$ & $(\mathrm{B})$ & 21 & 15 & 6 \\
$(9,4,5)$ & $(\mathrm{A})$ & 29 & 15 & 13 \\
$(9,4,5)$ & $(\mathrm{B})$ & 25 & 15 & 8 \\
$(8,6,5)$ & $(\mathrm{A})$ & 26 & 12 & 10 \\
$(8,6,5)$ & $(\mathrm{B})$ & 21 & 15 & 7 \\
$(9,6,5)$ & $(\mathrm{A})$ & 24 & 16 & 9 \\
$(9,6,5)$ & $(\mathrm{B})$ & 36 & 25 & 12 \\
\hline
\end{tabular}


tolerance, namely, $\left\|\boldsymbol{x}^{k+1}-\boldsymbol{x}^{k}\right\| \leq 10^{-1}$ (in $\mathrm{mm}^{2}$ ) is satisfied. This requires much more iterations compared with the procedure described above. In the iteration history, we select every iterate that satisfies the cardinality constraint approximately, i.e., that satisfies $l-\left|J_{0}^{k}\right| \leq n$. For every selected iterate, we run the post-processing, i.e., we solve problem (11) with specifying the set of vanishing nodes.

The computational results are listed in Table 5 , where $\tilde{K}$ and $\tilde{K}_{\mathrm{p}}$ are the number of iterations required before convergence and the number of iterates that approximately satisfy the cardinality constraint, respectively. For all the $\tilde{K}_{\mathrm{p}}$ iterates, the post-processing yields the same solution as the one reported in Table 3. In the case $\left(N_{X}, N_{Y}, n\right)=(9,2,5)$ with initial point (A), the ADMM does not converge after 100 iterates. In this iteration history, there exist 77 iterates satisfying the cardinality constraint approximately. From all of them, the post-processing generates the solution reported in Table 3. In a nutshell, the set of vanishing nodes does not change, even if the ADMM iterations are continued after the iterate at which our heuristic stopping criterion is satisfied.

For ease of comparison, the number of iterations reported in Table 3 is listed again as $K^{*}$ in Table 5. Namely, our stopping criterion reduces the number of iterations from $\tilde{K}$ to $K^{*}$, without changing the final output. It is worth noting that the solutions found at between the $\left(K^{*}+1\right)$ iterate and $\tilde{K}$ iterate do not necessarily satisfy the cardinality constraint with $\epsilon$ tolerance.

\subsection{On choice of initial points}

Since we apply ADMM to a nonconvex problem, the obtained solution may depend on the choice of initial points. In Section 6.1, we suggest to use the two initial points, (A) and (B), and adopt the better solution as the final output. In this section, we perform comparison with the results obtained by using randomly generated initial points to empirically justify our selection. As for two representative instances for which initial points (A) and (B) lead to different solution, we use $\left(N_{X}, N_{Y}, n\right)=(5,3,4)$ in section 6.1 and $\left(N_{X}, N_{Y}, n\right)=(9,4,5)$ in section 6.2 in the following numerical experiments.

As for randomly generated initial points, we examine two cases:

- Initial point $(\mathrm{C}): \boldsymbol{x}^{0}:=\left(V / \boldsymbol{c}^{\top} \boldsymbol{\xi}\right) \boldsymbol{\xi}, \boldsymbol{z}^{0}:=Z \boldsymbol{x}^{0}$, and $\boldsymbol{v}^{0}:=Z \boldsymbol{x}^{0}-\boldsymbol{z}^{0}=\mathbf{0}$, where $\boldsymbol{\xi} \in \mathbb{R}^{m}$ is a random vector with the entries drawn from $\mathcal{U}(0,1)$.

- Initial point (D): $\boldsymbol{x}^{0}:=\left(V / \boldsymbol{c}^{\top} \boldsymbol{\xi}\right) \boldsymbol{\xi}, \boldsymbol{z}^{0}:=Z \boldsymbol{x}^{0}$, and $\boldsymbol{v}^{0}:=\max \left\{z_{1}^{0}, \ldots, z_{l}^{0}\right\} \boldsymbol{\zeta}$, where $\boldsymbol{\xi} \in \mathbb{R}^{m}$ and $\boldsymbol{\zeta} \in \mathbb{R}^{l}$ are random vectors with the entries drawn from $\mathcal{U}(0,1)$.

We generate 100 samples of each of these initial points, and run our ADMM approach from every

Table 6: Computational results of ADMM from randomly generated initial points.

\begin{tabular}{llrrrr}
\hline$\left(N_{X}, N_{Y}, n\right)$ & Init. sol. & Min. $(\mathrm{J})$ & Max. $(\mathrm{J})$ & Mean $(\mathrm{J})$ & Var. $\left(\mathrm{J}^{2}\right)$ \\
\hline$(5,3,4)$ & $(\mathrm{C})$ & 5052.45 & 5052.45 & 5052.45 & 0.00 \\
$(5,3,4)$ & $(\mathrm{D})$ & 5007.41 & 5052.45 & 5049.74 & 115.56 \\
\hline$(9,4,5)$ & $(\mathrm{C})$ & 9000.00 & 9143.42 & 9039.78 & 3344.71 \\
$(9,4,5)$ & $(\mathrm{D})$ & 9000.00 & 9768.04 & 9076.47 & 13916.47 \\
\hline
\end{tabular}


sample. Table 6 reports the minimum value, maximum value, mean, and variance of the objective value.

For $\left(N_{X}, N_{Y}, n\right)=(5,3,4)$ with initial point $(\mathrm{C})$, in all the cases the ADMM converges to the same solution. This solution is the one obtained by using initial point (B), as shown in Table 2 , Therefore, using initial point (A) yielded a better solution (which is globally optimal) than using 100 samples of (C). In contrast, when initial point (D) was adopted, the global optimal solution is obtained from 7 sampled initial points, among 100 trials. From the other 93 samples, the ADMM converges to the solution obtained with initial point (B). The mean and the variance of the objective value are listed in Table 6. In this manner, it is demonstrated that the global optimal solution, easily obtained by carrying out our ADMM procedure with initial point (A), is rarely obtained from randomly generated initial points.

For $\left(N_{X}, N_{Y}, n\right)=(9,4,5)$ with initial point $(\mathrm{C})$, the best solution is same as the one obtained from initial point (B) in Table 3. This is not globally optimal. Among 100 trials, 67 sampled initial points yield this solution. In contrast, the objective value of the worst solution is larger than the one obtained from initial point (A). By using initial point (D), the variation of the objective value increased, but the global optimal solution was not obtained. Thus, the ADMM with randomly generated initial points could not find a solution better than the one obtained from initial point (B).

In short, for these two problem instances, using many randomly generated initial points does not yield a better solution. Therefore, using initial points (A) and (B) might be considered a reasonable selection.

\subsection{Application to robust optimization against uncertainty in external load}

The ADMM approach presented in this paper can be easily extended to the case in which the external load possesses uncertainty. The set of nodes at which the external forces can possibly be applied is supposed to be specified. Then we consider the robust optimization against the uncertainty, under the upper bound constraint on the number of nodes. In this section we examine efficiency of the ADMM applied to this problem, as an example of optimization problems that are not handled with current mainstream MIP solvers. The computation of this section was carried out on a $2.2 \mathrm{GHz}$ Intel Core i5 processor with 8 GB RAM.

As a concrete instance, consider the problem setting shown in Figure 3 . The external force is applied at the bottom right node, but this time its direction and magnitude are assumed to be uncertain. Without loss of generality, let $p_{1}$ and $p_{2}$ denote the horizontal and vertical components, respectively, of this external force. The set of possible realizations of the external load is defined by

$$
P=\left\{\left(p_{1}, p_{2}, 0, \ldots, 0\right)^{\top} \mid p_{1}=p_{1}^{0} \psi_{1}, p_{2}=p_{2}^{0} \psi_{2},\left\|\left(\psi_{1}, \psi_{2}\right)\right\| \leq 1\right\},
$$

where $p_{1}^{0}=30 \mathrm{kN}$ and $p_{2}^{0}=100 \mathrm{kN}$. With referring to 10$)$, we see that the compliance in the worst case is given by

$$
\hat{\pi}(\boldsymbol{x})=\sup \left\{2 \boldsymbol{p}^{\top} \boldsymbol{u}-\boldsymbol{u}^{\top} K(\boldsymbol{x}) \boldsymbol{u} \mid \boldsymbol{u} \in \mathbb{R}^{d}, \boldsymbol{p} \in P\right\} .
$$

In the following, we consider the minimization problem of this function. 
When the constraint on the number of nodes is not considered, it is known that this optimization problem can be recast as semidefinite programming (SDP) 6]. Since the upper bound constraint on the number of nodes is treated as presented in Section 3.2, the optimization problem under this constraint can be recast as mixed-integer semidefinite programming (MISDP). For comparison, we solve this MISDP problem with YALMIP [35], which finds a global optimal solution with a branchand-bound method [35]. We used YALMIP with the default setting, where SDP subproblems are solved with SeDuMi ver. 1.3 [47, 52]. Alternatively, consider the problem obtained by replacing the objective function of (14) by $\hat{\pi}$. It is fairly straightforward to apply the ADMM in Section 4 to this optimization problem. The subproblem solved to update the variable $\boldsymbol{x}$ at each iteration is formulated as SDP.

Table 7 reports the computational results. For five instances, $\left(N_{X}, N_{Y}, n\right)=(5,2,4),(5,3,4)$, $(5,4,4),(8,4,5)$, and $(9,4,5)$, the ADMM approach found the global optimal solutions. In all these cases, the computational cost of the ADMM is smaller than that of YALMIP. The difference of computational cost increases as the problem size increases. For $\left(N_{X}, N_{Y}, n\right)=(9,6,5)$, YALMIP did not terminate after 300 iterations. The best solution is same as the one found by the ADMM

Table 7: Computational results of the robust optimization with the cardinality constraint.

\begin{tabular}{|c|c|c|c|c|c|c|c|c|}
\hline \multirow[b]{2}{*}{$\left(N_{X}, N_{Y}, n\right)$} & \multicolumn{5}{|c|}{ ADMM } & \multicolumn{3}{|c|}{ MISDP } \\
\hline & Init. sol. & $w^{*}(\mathrm{~J})$ & $w^{*} / \bar{w}$ & \#iter & Time (s) & $\bar{w}(\mathrm{~J})$ & \#iter & Time (s) \\
\hline \multirow[t]{2}{*}{$(5,2,4)$} & $*(\mathrm{~A})$ & 12156.25 & 1.000 & 3 & 7.8 & 12156.25 & 22 & 11.8 \\
\hline & $*(\mathrm{~B})$ & 12156.25 & 1.000 & 3 & 6.7 & & & \\
\hline \multirow[t]{2}{*}{$(5,3,4)$} & $*(\mathrm{~A})$ & 5044.91 & 1.000 & 5 & 6.6 & 5044.91 & 9 & 7.6 \\
\hline & (B) & 5089.95 & - & 3 & 4.7 & & & \\
\hline \multirow[t]{2}{*}{$(5,4,4)$} & $*(\mathrm{~A})$ & 2840.63 & 1.000 & 2 & 6.7 & 2840.63 & 5 & 10.9 \\
\hline & $*(\mathrm{~B})$ & 2840.63 & 1.000 & 3 & 7.4 & & & \\
\hline \multirow[t]{2}{*}{$(8,2,5)$} & $*(\mathrm{~A})$ & 37605.63 & 1.071 & 11 & 16.5 & 35096.94 & 238 & 293.1 \\
\hline & $*(\mathrm{~B})$ & 37605.63 & 1.071 & 13 & 18.2 & & & \\
\hline \multirow[t]{2}{*}{$(9,2,5)$} & $*(\mathrm{~A})$ & 50101.25 & 1.070 & 20 & 32.0 & 46823.47 & 1484 & 2396.2 \\
\hline & $*(\mathrm{~B})$ & 50101.25 & 1.070 & 14 & 20.6 & & & \\
\hline \multirow[t]{2}{*}{$(8,4,5)$} & $*(\mathrm{~A})$ & 7076.25 & 1.000 & 11 & 67.0 & 7076.25 & 37 & 334.1 \\
\hline & $*(\mathrm{~B})$ & 7076.25 & 1.000 & 6 & 34.3 & & & \\
\hline \multirow[t]{2}{*}{$(9,4,5)$} & (A) & 9218.06 & - & 13 & 110.6 & & & \\
\hline & $*(\mathrm{~B})$ & 9050.63 & 1.000 & 6 & 47.0 & 9050.63 & 69 & 1026.2 \\
\hline \multirow[t]{2}{*}{$(8,6,5)$} & $*(\mathrm{~A})$ & 3317.84 & 1.037 & 10 & 118.8 & 3198.98 & 573 & 21992.7 \\
\hline & (B) & 26377.74 & - & 19 & 358.5 & & & \\
\hline \multirow[t]{2}{*}{$(9,6,5)$} & (A) & 4387.66 & - & 9 & 148.6 & & & \\
\hline & $*(\mathrm{~B})$ & 4255.40 & $\ddagger$ & 12 & 181.9 & $4387.66^{\ddagger}$ & $300^{\ddagger}$ & $16468.0^{\ddagger}$ \\
\hline
\end{tabular}




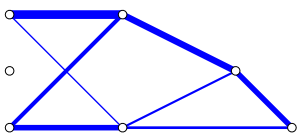

(a)

(b)

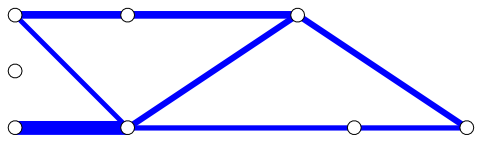

(d)

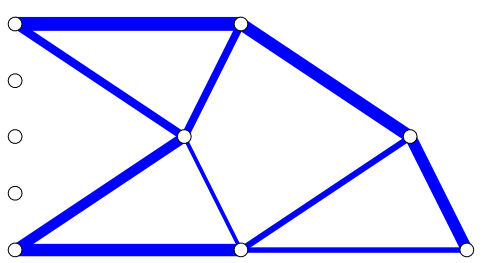

(f)

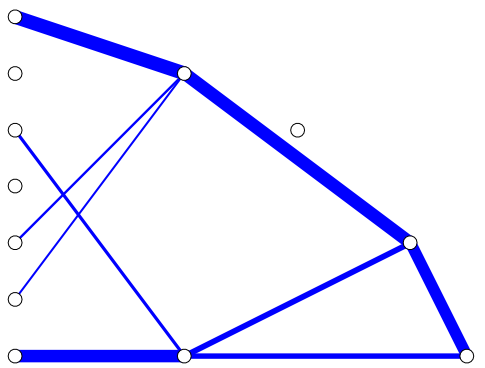

(h)
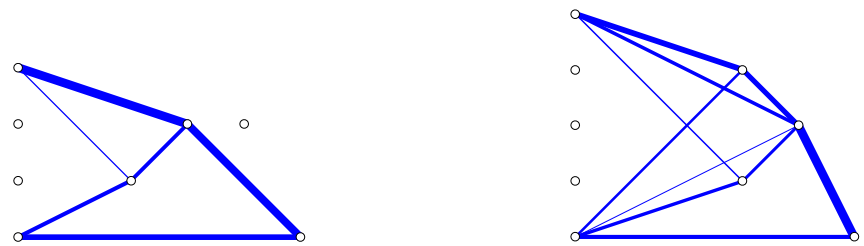

(c)

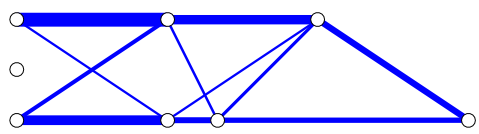

(e)

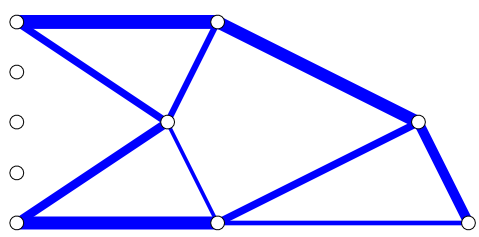

(g)

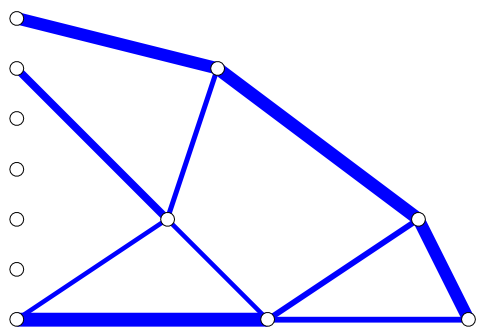

Figure 12: The solutions obtained by the ADMM applied to the robust optimization. (a) $\left(N_{X}, N_{Y}, n\right)=(5,2,4)$; (b) $\left(N_{X}, N_{Y}, n\right)=(5,3,4)$; (c) $\left(N_{X}, N_{Y}, n\right)=(5,4,4)$; (d) $\left(N_{X}, N_{Y}, n\right)=$ $(8,2,5) ;(\mathrm{e})\left(N_{X}, N_{Y}, n\right)=(9,2,5) ;(\mathrm{f})\left(N_{X}, N_{Y}, n\right)=(8,4,5) ;(\mathrm{g})\left(N_{X}, N_{Y}, n\right)=(9,4,5) ;(\mathrm{h})$ $\left(N_{X}, N_{Y}, n\right)=(8,6,5)$; and $(\mathrm{i})\left(N_{X}, N_{Y}, n\right)=(9,6,5)$.

with initial point (A), but a better solution was found by the ADMM with initial point (B). For three instances, $\left(N_{X}, N_{Y}, n\right)=(8,2,5),(9,2,5)$, and $(8,6,5)$, the solutions found by the ADMM are not optimal. The difference between the obtained objective value and the optimal value is $7 \%$ or less, like in the cases in section 6.3. The computational time required by YALMIP is more than 10 times (in some cases, more than 100 times) larger than that of the ADMM. Figure 12 collects the solutions obtained by the ADMM. The global optimal solutions that could not be obtained by the ADMM are shown in Figure 13 . The set of nodes in Figure 12(d) is much different from that in Figure 13(a) The solution in Figure 12(e) has only one node that is not included in the solution in Figure 13(b), Similarly, the difference between the solutions in Figure 12(h) and Figure 13(c) is the location of one node.

Figure 14 shows the solutions obtained by the ADMM for problem instances with larger sizes. The computational results are listed in Table 8. A global optimization method, YALMIP, cannot solve these problems within reasonable computational cost. 

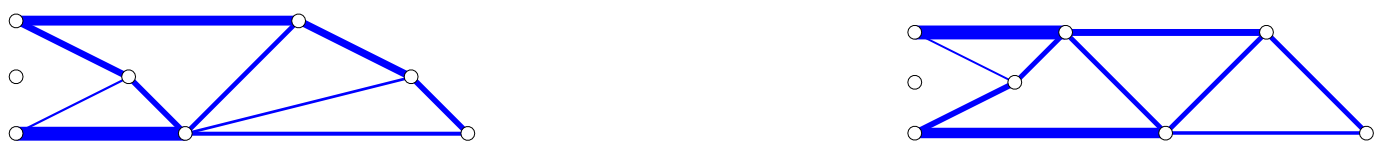

(a)

(b)

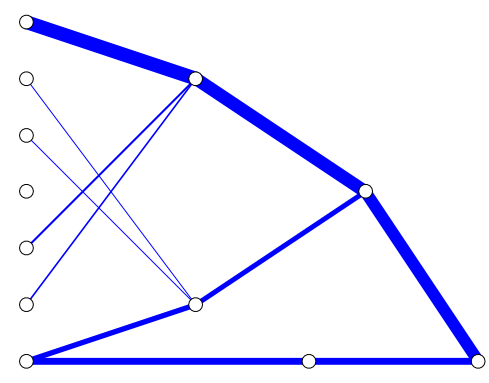

(c)

Figure 13: The optimal solutions of the robust optimization obtained by YALMIP. (a) $\left(N_{X}, N_{Y}, n\right)=(8,2,5) ;(\mathrm{b})\left(N_{X}, N_{Y}, n\right)=(9,2,5)$; and (c) $\left(N_{X}, N_{Y}, n\right)=(8,6,5)$.
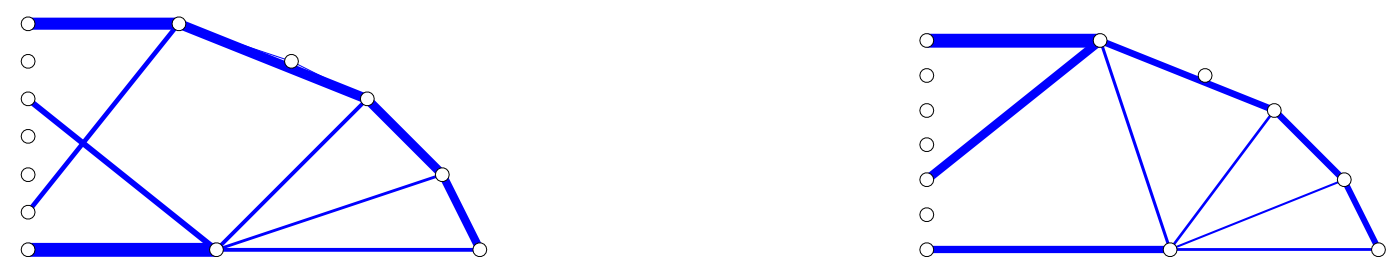

(a)

(b)

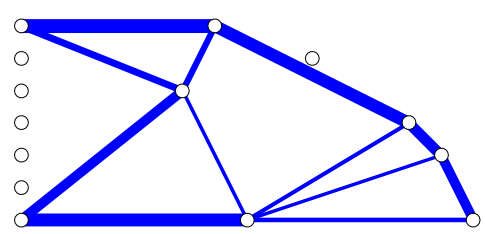

(c)

Figure 14: The solutions obtained by ADMM for the large-scale robust optimization problems. (a) $\left(N_{X}, N_{Y}, n\right)=(12,6,6) ;(\mathrm{b})\left(N_{X}, N_{Y}, n\right)=(13,6,6) ;(\mathrm{c})\left(N_{X}, N_{Y}, n\right)=(14,6,7)$;

Table 8: Computational results of the ADMM approach applied to the robust optimization with the cardinality constraint.

\begin{tabular}{lrrrr}
\hline$\left(N_{X}, N_{Y}, n\right)$ & Init. sol. & $w^{*}(\mathrm{~J})$ & \#iter & Time $(\mathrm{s})$ \\
\hline$(12,6,6)$ & $*(\mathrm{~A})$ & 7862.67 & 10 & 771.7 \\
& $(\mathrm{~B})$ & 9572.47 & 10 & 730.5 \\
\hline$(13,6,6)$ & $*(\mathrm{~A})$ & 10191.63 & 10 & 972.2 \\
& $(\mathrm{~B})$ & 13680.44 & 12 & 1108.4 \\
\hline$(14,6,7)$ & $*(\mathrm{~A})$ & 9772.59 & 9 & 1033.9 \\
& $(\mathrm{~B})$ & 14980.99 & 14 & 1614.1 \\
\hline
\end{tabular}




\section{Conclusions}

In this paper we have studied the compliance minimization of a truss with the limited number of nodes. It has been shown that this optimization problem can be formulated as the cardinalityconstrained SOCP. We have proposed a simple and efficient heuristic based on ADMM.

The problem considered in this paper can also be formulated as MISOCP involving the so-called big-M. In the numerical experiments, we have compared the proposed ADMM approach with a global optimization approach using the MISOCP formulation. For small-size problem instances, it has been confirmed that ADMM finds a global optimal solution. For middle-size instances, the objective value of the solution obtained by ADMM is often close to the optimal value. The number of iterations of ADMM is almost same for instances with different sizes. In contrast, the computational cost required by a standard MISOCP solver highly depends on instances, even if the instances have similar sizes.

In the numerical experiments, it has also been illustrated that, for some problem instances, the compliance minimization problem of a truss has some different optimal solutions, and the number of nodes can be decreased without losing the optimality. In most of the other cases, the number of nodes can be decreased at the expense of only small increase of the compliance.

Acknowledgments The work of the first author is partially supported by JSPS KAKENHI 15KT0109 and 17K06633.

\section{References}

[1] W. Achtziger: Local stability of trusses in the context of topology optimization. Part I: Exact modelling. Structural Optimization, 17, 235-246 (1999).

[2] E. D. Andersen, C. Roos, T. Terlaky: On implementing a primal-dual interior-point method for conic quadratic optimization. Mathematical Programming, 95, 249-277 (2003).

[3] M. F. Anjos, J. B. Lasserre (eds.): Handbook on Semidefinite, Conic and Polynomial Optimization. Springer, New York (2012).

[4] R. Arastoo, M. Bahavarnia, M. V. Kothare, N. Motee: Output feedback controller sparsification via $\mathcal{H}_{2}$-approximation. IFAC-PapersOnLine, 48, 112-117 (2015).

[5] A. Asadpoure, J. K. Guest, L. Valdevit: Incorporating fabrication cost into topology optimization of discrete structures and lattices. Structural and Multidisciplinary Optimization, 51, 385-396 (2015).

[6] A. Ben-Tal, A. Nemirovski: Robust truss topology optimization via semidefinite programming. SIAM Journal on Optimization, 7, 991-1016 (1997).

[7] A. Ben-Tal, A. Nemirovski: Lectures on Modern Convex Optimization: Analysis, Algorithms, and Engineering Applications. SIAM, Philadelphia (2001). 
[8] M. P. Bendsøe, A. Ben-Tal, J. Zowe: Optimization methods for truss geometry and topology design. Structural Optimization, 7, 141-159 (1994).

[9] D. Bertsimas, R. Shioda: Algorithm for cardinality-constrained quadratic optimization. Computational Optimization and Applications, 43, 1-22 (2009).

[10] S. Boyd, N. Parikh, E. Chu, B. Peleato, J. Eckstein: Distributed optimization and statistical learning via the alternating direction method of multipliers. Foundations and Trends in Machine Learning, 3, 1-122 (2010).

[11] A. M. Bruckstein, D. L. Donoho, M. Elad: From sparse solutions of systems of equations to sparse modeling of signals and images. SIAM Review, 51, 34-81 (2009).

[12] O. P. Burdakov, C. Kanzow, A. Schwartz: Mathematical programs with cardinality constraints: Reformulation by complementarity-type conditions and a regularization method. SIAM Journal on Optimimization, 26, 397-425 (2016).

[13] E. J. Candès, M. B. Wakin, S. P. Boyd: Enhancing sparsity by reweighted $\ell_{1}$ minimization. Journal of Fourier Analysis and Applications, 14, 877-905 (2008).

[14] R. Chartrand: Exact reconstruction of sparse signals via nonconvex minimization. IEEE Signal Processing Letters, 14, 707-710 (2007).

[15] R. Chartrand: Nonconvex splitting for regularized low-rank + sparse decomposition. IEEE Transactions on Signal Processing, 60, 5810-5819 (2012).

[16] R. Chartrand, B. Wohlberg: A nonconvex ADMM algorithm for group sparsity with sparse groups. 2013 IEEE International Conference on Acoustics, Speech and Signal Processing, Vancouver, pp. 6009-6013 (2013).

[17] X. T. Cui, X. J. Zheng, S. S. Zhu, X. L. Sun: Convex relaxations and MIQCQP reformulations for a class of cardinality-constrained portfolio selection problems. Journal of Global Optimization, 56, 1409-1423 (2013).

[18] S. Diamond, R. Takapoui, S. Boyd: A general system for heuristic minimization of convex functions over non-convex sets. Optimization Methods and Software, to appear. DOI:10.1080/ 10556788.2017 .1304548

[19] J. Gotoh, A. Takeda, K. Tono: DC formulations and algorithms for sparse optimization problems. Mathematical Programming, to appear. DOI:10.1007/s10107-017-1181-0

[20] M. Grant, S. Boyd: Graph implementations for nonsmooth convex programs. In: V. Blondel, S. Boyd, H. Kimura (eds.), Recent Advances in Learning and Control (A Tribute to M. Vidyasagar), Springer, pp. 95-110 (2008).

[21] M. Grant, S. Boyd: CVX: Matlab software for disciplined convex programming, version 2.1. http://cvxr.com/cvx/ (Accessed January 2017). 
[22] Gurobi Optimization, Inc.: Gurobi Optimizer Reference Manual. http://www.gurobi.com/ (Accessed September 2016).

[23] L. He, M. Gilbert: Rationalization of trusses generated via layout optimization. Structural and Multidisciplinary Optimization, 52, 677-694 (2015).

[24] X. Guo, G. D. Cheng, N. Olhoff: Optimum design of truss topology under buckling constraints. Structural and Multidisciplinary Optimization, 30, 169-180 (2005).

[25] G. A. Hegemier, W. Prager: On Michell trusses. International Journal of Mechanical Sciences, 11, 209-215 (1969).

[26] T. Kanamori, A. Takeda: Numerical study of learning algorithms on Stiefel manifold. Computational Management Science, 11, 319-340 (2014).

[27] Y. Kanno: Damper placement optimization in a shear building model with discrete design variables: A mixed-integer second-order cone programming approach. Earthquake Engineering and Structural Dynamics, 42, 1657-1676 (2013).

[28] Y. Kanno: Global optimization of trusses with constraints on number of different cross-sections: A mixed-integer second-order cone programming approach. Computational Optimization and Applications, 63, 203-236 (2016).

[29] Y. Kanno: Mixed-integer second-order cone programming for global optimization of compliance of frame structure with discrete design variables. Structural and Multidisciplinary Optimization, 54, 301-316 (2016).

[30] Y. Kanno, H. Yamada: A note on truss topology optimization under self-weight load: Mixedinteger second-order cone programming approach. Structural and Multidisciplinary Optimization, 56, 221-226 (2017).

[31] U. Kirsch: Optimal topologies of structures. Applied Mechanics Reviews (ASME), 42, 223-239 (1989).

[32] M. Kočvara: Truss topology design by linear conic optimization. In: T. Terlaky, M. F. Anjos, S. Ahmed (eds.), Advances and Trends in Optimization with Engineering Applications, SIAM, Philadelphia (to appear).

[33] H. A. Le Thi, T. Pham Dinh, H. M. Le, X. T. Vo: DC approximation approaches for sparse optimization. European Journal of Operational Research, 244, 26-46 (2015).

[34] F. Lin, M. Fardad, M. R. Jovanović: Design of optimal sparse feedback gains via the alternating direction method of multipliers. IEEE Transactions on Automatic Control, 58, 2426-2431 (2013).

[35] J. Löfberg: YALMIP: A toolbox for modeling and optimization in MATLAB. 2004 IEEE International Conference on Computer Aided Control System Design, Taipei, pp. 284-289 (2004). 
[36] S. Magnússon, M. G. Rabbat, C. Fischione: On the convergence of alternating direction Lagrangian methods for nonconvex structured optimization problems. IEEE Transactions on Control of Network Systems, 3, 296-309 (2016).

[37] E. Masazade, M. Fardad, P. K. Varshney: Sparsity-promoting extended Kalman filtering for target tracking in wireless sensor networks. IEEE Signal Processing Letters, 19, 845-848 (2012).

[38] A. Mazurek: Geometrical aspects of optimum truss like structures for three-force problem. Structural and Multidisciplinary Optimization, 45, 21-32 (2012).

[39] A. Mazurek, W. F. Baker, C. Tort: Geometrical aspects of optimum truss like structures. Structural and Multidisciplinary Optimization, 43, 231-242 (2011).

[40] K. Mela: Resolving issues with member buckling in truss topology optimization using a mixed variable approach. Structural and Multidisciplinary Optimization, 50, 1037-1049 (2014).

[41] A. G. A. Michell: The limits of economy of material in frame-structures. Philosophical Magazine, Series 6, 8, 589-597 (1904).

[42] R. Miyashiro, Y. Takano: Mixed integer second-order cone programming formulations for variable selection in linear regression. European Journal of Operational Research, 247, 721-731 (2015).

[43] B. K. Natarajan: Sparse approximate solutions to linear systems. SIAM Journal on Computing, 24, 227-234 (1995).

[44] M. Ohsaki: Optimization of Finite Dimensional Structures. CRC Press, Boca Raton (2011).

[45] M. Ohsaki, Y. Kanno, S. Tsuda: Linear programming approach to design of spatial link mechanism with partially rigid joints. Structural and Multidisciplinary Optimization, 50, 945-956 (2014).

[46] E. W. Parkes: Joints in optimum frameworks. International Journal of Solids and Structures, 11, 1017-1022 (1975).

[47] I. Pólik: Addendum to the SeDuMi User Guide: Version 1.1. Technical Report, Advanced Optimization Laboratory, McMaster University, Hamilton (2005). http://sedumi .ie.lehigh. edu/

[48] W. Prager: Optimal layout of cantilever trusses. Journal of Optimization Theory and Applications. 23, 111-117 (1977).

[49] W. Prager: Optimal layout of trusses with finite numbers of joints. Journal of the Mechanics and Physics of Solids, 26, 241-250 (1978).

[50] G. I. N. Rozvany: Difficulties in truss topology optimization with stress, local buckling and system stability constraints. Structural Optimization, 11, 213-217 (1996).

[51] G. Sagnol: PICOS: A Python Interface for Conic Optimization Solvers. http://picos.zib. de/ (Accessed February 2017). 
[52] J. F. Sturm: Using SeDuMi 1.02, a MATLAB toolbox for optimization over symmetric cones. Optimization Methods and Software, 11/12, 625-653 (1999).

[53] R. Takapoui, N. Moehle, S. Boyd, A. Bemporad: A simple effective heuristic for embedded mixed-integer quadratic programming. International Journal of Control, to appear. DOI:10. $1080 / 00207179.2017 .1316016$

[54] B. H. V. Topping: Shape optimization of skeletal structures: A review. Journal of Structural Engineering (ASCE), 109, 1933-1951 (1983).

[55] A. J. Torii, R. H. Lopez, L. F. F. Miguel: Design complexity control in truss optimization. Structural and Multidisciplinary Optimization, 54, 289-299 (2016).

[56] R. H. Tütüncü, K. C. Toh, M. J. Todd: Solving semidefinite-quadratic-linear programs using SDPT3. Mathematical Programming, B95, 189-217 (2003).

[57] X. Zheng, X. Sun, D. Li, J. Sun: Successive convex approximations to cardinality-constrained convex programs: A piecewise-linear DC approach. Computational Optimization and Applications, 59, 379-397 (2014). 